\title{
Relativistic and pseudorelativistic formulation of nonlinear envelope equations with spatiotemporal dispersion. I. Cubic-quintic systems
}

\author{
J. M. Christian,* G. S. McDonald, and A. Kotsampaseris \\ Joule Physics Laboratory, School of Computing, Science and Engineering, \\ University of Salford, Greater Manchester M5 4WT, United Kingdom
}

(Dated: July 26, 2018)

\begin{abstract}
A generic envelope equation is proposed for describing the evolution of scalar pulses in systems with spatiotemporal dispersion and cubic-quintic nonlinearity. Our analysis has application, for instance, in waveguide optics where the physical origin of the dielectric response lies in the $\chi^{(3)}$ and $\chi^{(5)}$ susceptibilities. Exact analytical bright and gray solitons are derived by coordinate transformations and methods of direct integration. Known solitons of conventional pulse theory (based on nonlinear-Schrödinger prescriptions) are shown to emerge asymptotically as subsets of the more general spatiotemporal solutions, and simulations test the stability of the latter through a class of perturbed initial-value problem.
\end{abstract}

PACS numbers: 42.65.-k, 42.65.Fs, 42.65.Tg, 42.65.Wi, 05.45.Yv

Keywords: bright solitons, dark solitons, Kerr effect, spatial dispersion, waveguide optics

\section{INTRODUCTION}

Understanding the formation, propagation, and interactions between solitons is a fundamental objective in many branches of nonlinear science $[1,2]$. These selflocalizing and self-stabilizing wavepackets are elementary excitations that may emerge in essentially any system possessing both linear and nonlinear dispersive elements [3]. Since solitons and solitary-wave phenomena are frequently described by amplitude equations that anticipate slowly-varying wave envelopes, a key question to address is their properties beyond this prevailing (if often justifiable) level of approximation.

In a set of earlier papers, we proposed [4] and analyzed [5, 6] a model for describing the longitudinal evolution of scalar wavepackets in systems with linear dispersion [both temporal (group-velocity) and spatial forms] and cubic nonlinearity. The governing equation was not bound by the ubiquitous slowly-varying envelope approximation (SVEA), and its structure was thus rendered fully-second-order in laboratory time $t$ (the coordinate in which pulses are typically localized) and space $z$ (the evolution coordinate). A consequence of deploying such a symmetrized model is that a Galilean boost to local-time coordinates $z_{\mathrm{loc}} \equiv z$ and $t_{\mathrm{loc}} \equiv t-z / v_{g}$ (which define a reference frame moving relative to the laboratory at group velocity $v_{g}$ in the $+z$ direction) obscures the equal status of space and time: when expressed in terms of derivatives with respect to $z_{\text {loc }}$ and $t_{\text {loc }}$, the transformed wave equation is not interpreted quite so intuitively due to the appearance of a mixed partial differential operator $\partial^{2} / \partial z_{\text {loc }} \partial t_{\text {loc }}[7]$.

The Galilean-boost procedure may be safely discarded because its introduction is, in any case, arbitrary and usually made for convenience [4]. Without it, and in

\footnotetext{
* Corresponding author: j.christian@salford.ac.uk
}

the absence of the SVEA, a compact framework emerges for modelling pulse phenomena in wave-based systems with spatial and temporal dispersion. The spirit of our more geometric formalism (viz., frame-of-reference considerations, transformations in the space-time plane, covariance of the wave equation, invariant quantities, and Lorentz-like combination rules for velocities) has strong connections to Einstein's special theory of relativity [8]. Moreover, all the results of conventional pulse theory are recoverable asymptotically (when simultaneous multiparameter expansions are applied to the spatiotemporal solutions) in much the same way as Newtonian dynamics appears in the low speed limit of relativistic mechanics.

The spatiotemporal description of wave propagation is rather general. Previously, we have applied it in the arena of waveguide optics when the nonlinear polarization of the host medium is dominated by the $\chi^{(3)}$ susceptibility [9]. Such a simple configuration has been studied extensively for over four decades [10] through the prism of slowly-varying envelopes and Galilean boosts, with many classic analyses based on nonlinear Schrödinger (NLS) equations [11-13]. With space-time symmetry firmly in mind, it may be seen that the seminal work of Biancalana and Creatore [14] can play an important role in certain physical regimes. They identified that in some semiconductors (e.g., ZnCdSe/ZnSe superlattices), spatial material dispersion (an effect connected to photonexciton coupling [15]) may be described by a contribution to the envelope equation that is proportional to the second longitudinal derivative, $\partial^{2} / \partial z^{2}$. Related phenomena cannot be adequately described within the SVEA, and $\partial^{2} / \partial z^{2}$ considerations hence underpin modern contexts for research into new classes of generic relativistic- and pseudorelativistic-type propagation problems [4].

Here, we generalize our earlier analyses from cubic [46 ] to cubic-quintic systems. In wave optics, the quintic term might arise from excitation of the higher-order $\chi^{(5)}$ susceptibility. The combined $\chi^{(3)}-\chi^{(5)}$ response, proposed by Pushkarov et al. [16], has come to play an 
important role in photonics and is crucial for modelling a wide range of materials: liquid carbon disulfide [17], ultraviolet-grade fused silica [18], AlGaAs semiconductors operating just below the half bandgap [19], some semiconductor-doped glasses [20, 21], the polydiaceteylene para-toluene sulfonate $\pi$-conjugated polymer [22], chalcogenide glasses [23], and some transparent organic materials [24].

Decades after its proposal, the cubic-quintic nonlinearity continues to pique the interest of researchers. For instance, Stegeman et al. [25] have provided an in-depth analysis of the tensor character of $\chi^{(5)}$ in order to accurately quantify constitutive relations in optical materials beyond the well-understood Kerr regime. More recently, Besse et al. [26] generalized the standard Lorentz model (routinely used for introducing phenomenological descriptions of nonlinear dynamical effects [9]) to account for a sextic term in the potential energy well of a onedimensional oscillator.

The conventional cubic-quintic envelope equation [in its equivalent spatial (beam) and temporal (pulse) guises] has well-known exact analytical solutions, principally the bright soliton of Gatz and Herrmann [27] and its dark counterpart derived by Herrmann [28] (both of which are exponentially-localized states). Gagnon [29] and others [30] have considered a broader spectrum of solutions (including antidark solitons, partially-delocalized amplitude kinks, and cnoidal waves) that may exist depending upon the interplay between group-velocity dispersion (GVD) and nonlinearity. The stability of, and interactions between, these excitations have been addressed through detailed simulations [31]. Cubic-quintic models also admit the possibility of algebraic solitons (weaklylocalized states with slower power-law asymptotics that correspond to a boundary separating localized hyperbolic excitations and periodic wavetrains) [32].

The layout of this paper is as follows. In Sec. II, the dimensionless cubic-quintic spatiotemporal model is introduced and a generic separation-of-variables technique is deployed to derive a pair of coupled equations describing the intensity and phase quadratures of an arbitrary solution. The properties of key operator combinations used throughout the analysis are also discussed. In Secs. III and IV, we derive exact bright and dark (gray) solitons by direct integration of the quadrature equations subject to appropriate boundary conditions (families of algebraic and amplitude-kink waves are presented in appendices $\mathrm{A}$ and $\mathrm{B}$, respectively). More general solutions accommodating a finite frequency shift are detailed in Sec. V, which are arrived at on the basis of coordinate transformations. Asymptotic analysis in Sec. VI demonstrates the recovery of known solitons in the limit of slowlyvarying envelopes (a feature that is required both physically and mathematically), and numerical simulations test the robustness of spatiotemporal solitons via a class of perturbed initial-value problem in Sec. VII. We conclude, in Sec. VIII, with comments about the potential applications of our work.

\section{SPATIOTEMPORAL MODEL}

\section{A. Envelope equation}

As an example, we consider a cw electric field defined by $E(t, z)=A(t, z) \exp \left[i\left(k_{0} z-\omega_{0} t\right)\right]+$ c.c., where "c.c." denotes complex conjugation of the preceding quantity, $\omega_{0}$ and $k_{0}=n_{0} \omega_{0} / c$ are the angular frequency and propagation constant, respectively, for a wave travelling in a host medium with linear refractive index $n_{0} \equiv n_{0}\left(\omega_{0}\right)$, and $c$ is the vacuum speed of light. By adopting the standard Fourier decomposition to accommodate leadingorder temporal dispersion [9, 33], the complex amplitude $A(t, z)$ can be shown to satisfy the following envelope equation that is symmetric in space and time:

$$
\begin{aligned}
\frac{1}{2 k_{0}} \frac{\partial^{2} A}{\partial z^{2}}+i\left(\frac{\partial A}{\partial z}\right. & \left.+k_{1} \frac{\partial A}{\partial t}\right)-\frac{k_{2}}{2} \frac{\partial^{2} A}{\partial t^{2}} \\
& +\frac{\omega_{0}}{c}\left(n_{2}|A|^{2}+n_{4}|A|^{4}\right) A=0
\end{aligned}
$$

Here, $k_{1} \equiv(\partial k / \partial \omega)_{\omega_{0}}=1 / v_{g}$ is the inverse of the group velocity $v_{g}$ and $k_{2} \equiv\left(\partial^{2} k / \partial \omega^{2}\right)_{\omega_{0}}$ the GVD coefficient, where $k$ is related to the mode eigenvalue (obtained by solving Maxwell's equations for the transverse distribution of the guided field [33]). Coefficients $n_{2}$ and $n_{4}$ are directly related to the third- and fifth-order susceptibilities $[17,25,26]$. The self-induced refractive-index change $n_{\mathrm{NL}}\left(|A|^{2}\right)$, well-described by $n_{\mathrm{NL}} \equiv n_{2}|A|^{2}+n_{4}|A|^{4}$ in scalar cubic-quintic regimes, is then assumed to be a small perturbation compared to the dominant linear part $n_{0}$ (and, typically, $n_{4}|A|^{4}$ is much weaker than $n_{2}|A|^{2}$ in these contexts) [16].

With reference to a conventional Gaussian pulse of fullwidth $2 t_{p}$ and dispersion length $L=t_{p}^{2} /\left|k_{2}\right|[9,33]$, one can introduce dimensionless coordinates $\zeta \equiv z / L$ and $\tau \equiv$ $t / t_{p}$. By substituting $A(\tau, \zeta)=A_{0} u(\tau, \zeta)$ into Eq. (1), a governing equation for the dimensionless envelope $u$ may be obtained:

$\kappa \frac{\partial^{2} u}{\partial \zeta^{2}}+i\left(\frac{\partial u}{\partial \zeta}+\alpha \frac{\partial u}{\partial \tau}\right)+\frac{s}{2} \frac{\partial^{2} u}{\partial \tau^{2}}+\gamma_{2}|u|^{2} u+\gamma_{4}|u|^{4} u=0$,

where $\alpha=k_{1} t_{p} /\left|k_{2}\right|$ is a ratio of group speeds and $s=$ $-\operatorname{sgn}\left(k_{2}\right)= \pm 1$ flags the sign of the GVD coefficient $(+1$ for anomalous, -1 for normal). When the electric field is measured in units of $A_{0}=\left(n_{0} /\left|n_{2}\right| k_{0} L\right)^{1 / 2}$, it follows that $\gamma_{2}=\operatorname{sgn}\left(n_{2}\right)$ while $\gamma_{4}=n_{4} A_{0}^{2} /\left|n_{2}\right|$ parametrizes the strength of quintic to cubic nonlinear phase shifts. Note that by setting $\alpha=0$ and interpreting $\tau$ as a (normalized) transverse spatial coordinate, Eq. (2) is formally identical to the scalar Helmholtz equation describing bright [34] and dark [35] cw beams in two-dimensional cubic-quintic systems. The propagation contribution to spatial dispersion, arising from the confined electromagnetic mode, is parametrized by $\kappa \equiv 1 / 2 k_{0} L=c\left|k_{2}\right| / 2 n_{0} \omega_{0} t_{p}^{2} \ll \mathcal{O}(1)$ [4]; the material contribution [14] can be included within the definition of $\kappa$ to give a lumped parameter which we take to be positive here without loss of generality. 
Since $\kappa \partial^{2} u / \partial \zeta^{2}$ is potentially small, it is tempting to either neglect it completely (the essence of the SVEA) or, slightly more satisfactorily, consider it as an $\mathcal{O}(\kappa)$ perturbation using a generalization of the methods applied to cw beams in cubically-nonlinear systems [36]. Such an approach is unnecessary and actually increases model complexity. As we will show, Eq. (2) can be treated exactly (i.e., without further approximation).

\section{B. General quadrature equations}

We begin by seeking solutions to Eq. (2) that can be represented by the Madelung-type ansatz

$$
u(\tau, \zeta)=\rho^{1 / 2}(\tau, \zeta) \exp [i \psi(\tau, \zeta)]
$$

where $\rho(\tau, \zeta)$ and $\psi(\tau, \zeta)$ are the intensity and (total) phase quadratures, respectively (and, hence, are taken to be real functions). By substituting the decomposition for $u$ into Eq. (2) and collecting the real and imaginary parts, one obtains

$$
\begin{gathered}
\frac{2}{\rho}\left(\frac{\partial^{2} \rho}{\partial \tau^{2}}+2 s \kappa \frac{\partial^{2} \rho}{\partial \zeta^{2}}\right)-\frac{1}{\rho^{2}}\left[\left(\frac{\partial \rho}{\partial \tau}\right)^{2}+2 s \kappa\left(\frac{\partial \rho}{\partial \zeta}\right)^{2}\right] \\
-4\left[\left(\frac{\partial \psi}{\partial \tau}\right)^{2}+2 s \kappa\left(\frac{\partial \psi}{\partial \zeta}\right)^{2}\right] \\
-8 s\left[\left(\frac{\partial \psi}{\partial \zeta}+\alpha \frac{\partial \psi}{\partial \tau}\right)-\left(\gamma_{2}+\gamma_{4} \rho\right) \rho\right]=0
\end{gathered}
$$

and

$$
\begin{aligned}
& \rho\left(\frac{\partial^{2} \psi}{\partial \tau^{2}}+2 s \kappa \frac{\partial^{2} \psi}{\partial \zeta^{2}}\right)+\left(\frac{\partial \psi}{\partial \tau} \frac{\partial \rho}{\partial \tau}+2 s \kappa \frac{\partial \psi}{\partial \zeta} \frac{\partial \rho}{\partial \zeta}\right) \\
& +s\left(\frac{\partial \rho}{\partial \zeta}+\alpha \frac{\partial \rho}{\partial \tau}\right)=0
\end{aligned}
$$

respectively. These equations are somewhat symmetrical in $\rho$ and $\psi$ derivatives, being a direct spatiotemporal generalization of those typically considered in conventional pulse theory. They can be expressed in a more convenient form by eliminating the longitudinal rapid-phase contribution associated with the background carrier wave according to $\psi(\tau, \zeta) \equiv \Psi(\tau, \zeta)-\zeta / 2 \kappa$. It follows that $\rho$ and $\Psi$ are then coupled through

$$
\begin{gathered}
\frac{2}{\rho}\left(\frac{\partial^{2} \rho}{\partial \tau^{2}}+2 s \kappa \frac{\partial^{2} \rho}{\partial \zeta^{2}}\right)-\frac{1}{\rho^{2}}\left[\left(\frac{\partial \rho}{\partial \tau}\right)^{2}+2 s \kappa\left(\frac{\partial \rho}{\partial \zeta}\right)^{2}\right] \\
-4\left[\left(\frac{\partial \Psi}{\partial \tau}\right)^{2}+2 s \kappa\left(\frac{\partial \Psi}{\partial \zeta}\right)^{2}\right] \\
-8 s\left[\alpha \frac{\partial \Psi}{\partial \tau}-\frac{1}{4 \kappa}-\left(\gamma_{2}+\gamma_{4} \rho\right) \rho\right]=0
\end{gathered}
$$

and

$$
\begin{aligned}
& \rho\left(\frac{\partial^{2} \Psi}{\partial \tau^{2}}+2 s \kappa \frac{\partial^{2} \Psi}{\partial \zeta^{2}}\right)+\left(\frac{\partial \Psi}{\partial \tau} \frac{\partial \rho}{\partial \tau}+2 s \kappa \frac{\partial \Psi}{\partial \zeta} \frac{\partial \rho}{\partial \zeta}\right) \\
& \quad+s \alpha \frac{\partial \rho}{\partial \tau}=0
\end{aligned}
$$

To find particular (i.e., soliton) solutions, Eqs. (4a) and (4b) must be supplemented by appropriate boundary conditions on $\rho$ and $\Psi$.

\section{Space-time coordinate transformation}

Analysis is most easily facilitated by introducing a lumped space-time coordinate $\xi \equiv \xi(\tau, \zeta)$, defined as

$$
\xi(\tau, \zeta) \equiv \frac{\tau-V_{0} \zeta}{\sqrt{1+2 s \kappa V_{0}^{2}}}
$$

One might interpret $\xi$ as a time coordinate in the rest frame of the pulse under consideration (that is, in the frame where the pulse is stationary) [4]. Although the status of $V_{0}$ corresponds to a velocity-like parameter in the theory of beams $[34,35]$, in the context of pulses it is, strictly, related to the inverse velocity in unscaled units.

The advantage of introducing $\xi$ is that it allows one to simplify combinations of partial derivatives. On the one hand, operators $\partial / \partial \tau$ and $\partial / \partial \zeta$ may be recast as

$$
\frac{\partial}{\partial \tau}=\frac{1}{\sqrt{1+2 s \kappa V_{0}^{2}}} \frac{d}{d \xi} \text { and } \quad \frac{\partial}{\partial \zeta}=-\frac{V_{0}}{\sqrt{1+2 s \kappa V_{0}^{2}}} \frac{d}{d \xi}
$$

On the other hand, combinations of operators appearing in Eqs. (4a) and (4b) transform according to

$$
\frac{\partial \cdot \partial \cdot}{\partial \tau} \frac{\partial}{\partial \tau}+2 s \kappa \frac{\partial \cdot \partial \cdot}{\partial \zeta} \frac{d \cdot d \cdot}{\partial \zeta}=\frac{d}{d \xi} \frac{}{d \xi}
$$

and

$$
\left(\frac{\partial^{2}}{\partial \tau^{2}}+2 s \kappa \frac{\partial^{2}}{\partial \zeta^{2}}\right) \cdot=\frac{d^{2}}{d \xi^{2}}
$$

where " ." symbolizes a place reserver. With careful deployment of transformation (5a)-(5d), the quadrature equations in both spatiotemporal and conventional [27, 28] formalisms can be shown to map onto each other in an essential way. For example, the functional form of pulse shapes is determined by the interplay between dispersion and nonlinearity, and should not be dependent upon the choice of reference frame.

\section{BRIGHT SOLITON PULSES}

We begin our analysis of solitary states by considering bright solitons (bell-shaped profiles that exist on top of a modulationally-stable zero-amplitude background wave). These solutions may be expected to possess an intrinsic velocity proportional to $\alpha$ since they are moving with respect to the (stationary) waveguide. Throughout the rest of the paper, we denote the intensity distribution by $\rho_{\mathrm{b}}(\tau, \zeta)$. The phase has a more subtle decomposition. 


\section{A. Symmetry reduction}

In the anomalous dispersion regime (where $s=+1$ ), Eqs. (4a) and (4b) can be integrated exactly. By setting $\Psi(\tau, \zeta)=\Psi_{\mathrm{b}}(\tau, \zeta)+K_{\mathrm{b}} \zeta$, where $K_{\mathrm{b}}$ is the soliton propagation constant and $\Psi_{\mathrm{b}}=0$ (so there is no phase change across the temporal extent of the wavepacket), one can show that $\rho_{\mathrm{b}}$ must satisfy the pair of simultaneous equations:

$$
\begin{gathered}
\frac{2}{\rho_{\mathrm{b}}}\left(\frac{\partial^{2} \rho_{\mathrm{b}}}{\partial \tau^{2}}+2 \kappa \frac{\partial^{2} \rho_{\mathrm{b}}}{\partial \zeta^{2}}\right)-\frac{1}{\rho_{\mathrm{b}}^{2}}\left[\left(\frac{\partial \rho_{\mathrm{b}}}{\partial \tau}\right)^{2}+2 \kappa\left(\frac{\partial \rho_{\mathrm{b}}}{\partial \zeta}\right)^{2}\right] \\
-8\left(\kappa K_{\mathrm{b}}^{2}-\frac{1}{4 \kappa}\right)+8\left(\gamma_{2}+\gamma_{4} \rho_{\mathrm{b}}\right) \rho_{\mathrm{b}}=0 \\
\alpha \frac{\partial \rho_{\mathrm{b}}}{\partial \tau}+2 \kappa K_{\mathrm{b}} \frac{\partial \rho_{\mathrm{b}}}{\partial \zeta}=0
\end{gathered}
$$

According to the transformation detailed in Sec. II, where $s=+1$ and the velocity parameter is labelled as $V_{0 \mathrm{~b}}$, Eqs. (6a) and (6b) simplify to

$$
\begin{gathered}
\frac{d}{d \rho_{\mathrm{b}}}\left[\frac{1}{\rho_{\mathrm{b}}}\left(\frac{d \rho_{\mathrm{b}}}{d \xi}\right)^{2}\right]=8\left[\beta_{\mathrm{b}}-\left(\gamma_{2}+\gamma_{4} \rho\right) \rho_{\mathrm{b}}\right], \\
\left(\alpha-2 \kappa K_{\mathrm{b}} V_{0 \mathrm{~b}}\right) \frac{d \rho_{\mathrm{b}}}{d \xi}=0 .
\end{gathered}
$$

Equation (7a) is parametrized by $\beta_{\mathrm{b}} \equiv \kappa K_{\mathrm{b}}^{2}-1 / 4 \kappa$, which is quadratic in $K_{\mathrm{b}}$ and thus yields two branches: $K_{\mathrm{b}}= \pm\left(1+4 \kappa \beta_{\mathrm{b}}\right)^{1 / 2} / 2 \kappa$, where the $+(-)$ sign describes wavepackets travelling in the forward (backward) longitudinal sense.

\section{B. Intensity quadrature}

Direct integration of Eq. (7a) with respect to $\rho_{\mathrm{b}}$ yields

$$
\left(\frac{d \rho_{\mathrm{b}}}{d \xi}\right)^{2}=-4\left(\gamma_{2}+\frac{2}{3} \gamma_{4} \rho_{\mathrm{b}}\right) \rho_{\mathrm{b}}^{3}+8 \beta_{\mathrm{b}} \rho_{\mathrm{b}}^{2}+c_{2 \mathrm{~b}} \rho_{\mathrm{b}}
$$

where $c_{2} \mathrm{~b}$ is a constant to be determined from the solution boundary conditions. As $\xi \rightarrow \pm \infty$, one has that $\rho_{\mathrm{b}} \rightarrow 0$ and $\left(d \rho_{\mathrm{b}} / d \xi\right)^{2} \rightarrow 0$. Applying these conditions to Eq. (8a) shows that $c_{2 \mathrm{~b}}=0$. Similarly, when $\xi \rightarrow 0$, one has that $\rho_{\mathrm{b}} \rightarrow \rho_{0}$ and $\left(d \rho_{\mathrm{b}} / d \xi\right)^{2} \rightarrow 0$, giving rise to

$$
\beta_{\mathrm{b}} \equiv\left(\gamma_{2}+\frac{2}{3} \gamma_{4} \rho_{0}\right) \frac{\rho_{0}}{2}
$$

The physical interpretation of $\beta_{\mathrm{b}}$ will become apparent later on. To facilitate a second integration, it is convenient to factorize the right-hand side of Eq. (8a) so that

$$
\left(\frac{d \rho_{\mathrm{b}}}{d \xi}\right)^{2}=4 \rho_{\mathrm{b}}^{2}\left(\rho_{0}-\rho_{\mathrm{b}}\right)\left(g_{1 \mathrm{~b}} \rho_{\mathrm{b}}+g_{0 \mathrm{~b}}\right)
$$

where $g_{1 \mathrm{~b}} \equiv(2 / 3) \gamma_{4}$ and $g_{0 \mathrm{~b}} \equiv \gamma_{2}+(2 / 3) \gamma_{4} \rho_{0}$. Separation of Eq. (8c) and deployment of a standard integral results in the intensity quadrature

$$
\rho_{\mathrm{b}}(\xi)=\frac{4 \beta_{\mathrm{b}}}{B \cosh \left(2 \sqrt{2 \beta_{\mathrm{b}}} \xi\right)+\gamma_{2}},
$$

where $B \equiv\left[\gamma_{2}^{2}+(16 / 3) \gamma_{4} \beta_{\mathrm{b}}\right]^{1 / 2}$. The solution is selfconsistent since $\rho(0)=4 \beta_{\mathrm{b}} /\left(B+\gamma_{2}\right) \equiv \rho_{0}$ also gives rise to the result for $\beta_{\mathrm{b}}$ in Eq. (8b). We also note that $B$ can be expressed as a function of $\rho_{0}$, such that $B=$ $\gamma_{2}+(4 / 3) \gamma_{4} \rho_{0}$. The existence of a localized bell-shaped solution requires both $\beta_{\mathrm{b}}>0$ and $B>0$.

We note that the spatiotemporal intensity profile in its rest frame [see Eq. (9)] maps directly onto the solution derived by Gatz and Herrmann [27] in the local-time frame, as it must. Such a result is not altogether surprising mathematically since we have deliberately constructed a coordinate transformation [c.f. Eq. (5a)] to draw out such a symmetry. In terms of a fundamental physical principle, the form of the pulse shape must be insensitive to the coordinate system one chooses (since, as mentioned previously, any such choice is arbitrary). Linear boosts to take observers between different frames of reference result in a contraction or dilation of the projected pulse width but these geometrical operations cannot change its structure [4-6].

\section{Intrinsic velocity}

In order for Eq. (7b) to hold for arbitrary gradients $d \rho_{\mathrm{b}} / d \xi$, it must be that $\alpha-2 \kappa K_{\mathrm{b}} V_{0 \mathrm{~b}}=0$ or, equivalently, $V_{0 \mathrm{~b}}=\alpha / 2 \kappa K_{\mathrm{b}}$. Substituting for $K_{\mathrm{b}}$ then gives rise to

$$
V_{0 \mathrm{~b}}= \pm \frac{\alpha}{\sqrt{1+4 \kappa \beta_{\mathrm{b}}}}
$$

Later it will be convenient to release the \pm sign (which is determined by the longitudinal propagation sense) directly into the argument of the cosh function $[5,6]$.

Equation (10) reveals that pulse-type solutions to Eq. (2) are associated with an intrinsic velocity parameter that has a weak dependence on the peak intensity. Since $\beta_{\mathrm{b}}$ increases with $\rho_{0}$, one may conclude that pulse speeds in the laboratory frame (which are proportional to $1 / V_{0 \mathrm{~b}}$ ) increase with $\rho_{0}$. In contrast, solitons (and, more generally, arbitrarily-shaped pulses) of conventional NLS-type theory [i.e., Eq. (2) in the absence of the first term] do not tend to exhibit such a nonlinear phenomenon although amplitude-dependent speeds are common in other universal wave equations (such as that of Korteweg and de Vries [1]).

Finally, we address existence criteria. For purelypositive nonlinearity coefficients, the solution continuum has $\beta_{\mathrm{b}}>0$ for all $\rho_{0}>0$ while no bell-shaped solution exists in the purely-negative case. For the competing nonlinearity $\gamma_{2}>0$ and $\gamma_{4}<0$, it follows from $B>0$ (the dominant inequality) that $0<\beta_{\mathrm{b}}<\beta_{\mathrm{b} \max }$, 
where $\beta_{\mathrm{b} \max }=(3 / 16) \gamma_{2}^{2} / \gamma_{4}$. Hence, from Eq. (8b), there exists a maximum peak intensity $\rho_{0 \max }$ such that $0<\rho_{0}<\rho_{0 \max } \equiv(3 / 4) \gamma_{2} /\left|\gamma_{4}\right|$. In the complementary regime $\gamma_{2}<0$ and $\gamma_{4}>0$, solutions with $\beta_{\mathrm{b}}>0$ possess a minimum intensity $\rho_{0 \text { min }}$ determined from the inequality $\rho_{0}>\rho_{0 \min } \equiv(3 / 2)\left|\gamma_{2}\right| / \gamma_{4}$.

\section{DARK SOLITON PULSES}

We now turn our attention to dark solitons (whose intensity and phase quadratures are denoted by $\rho_{\mathrm{d}}$ and $\Psi_{\mathrm{d}}$, respectively) which comprise a phase-topological gray 'dip' travelling across a cw background whose stability against any such disturbance is crucial for ensuring the existence of the localized state. Attention is thus first paid to $\mathrm{cw}$ modulational instability (MI).

\section{A. Continuous-wave solutions}

The cw solutions of Eq. (2) are those states $u_{\mathrm{cw}}$ that are uniform in space and time:

$$
u_{\mathrm{cw}}(\tau, \zeta)=\rho_{0}^{1 / 2} \exp \left[i\left(-\Omega \tau+K_{\mathrm{cw}} \zeta\right)\right] \exp \left(-i \frac{\zeta}{2 \kappa}\right),
$$

where $\left|u_{\mathrm{cw}}\right|^{2}=\rho_{0}$ is the wave intensity, $\Omega$ represents a frequency shift (treated here as a free parameter), $K_{\mathrm{cw}}=$ $\pm\left[1+4 \kappa \beta_{\mathrm{cw}}+4 \kappa \Omega(\alpha-s \Omega / 2)\right]^{1 / 2} / 2 \kappa$ is the propagation constant, and $\beta_{\mathrm{cw}} \equiv\left(\gamma_{2}+\gamma_{4} \rho_{0}\right) \rho_{0}$.

Applying a generalization of the perturbative method developed in Ref. [6] to Eq. (2), we disturb $u$ by a small amount and derive a linearized equation describing the short-term evolution of the perturbation field. One then seeks Fourier mode solutions of that linear problem at frequency $\Omega_{\mathrm{p}}$, whereupon it can be shown that cw solution (11a) becomes unstable against long-wavelength modulations whenever

$$
\frac{\Omega_{\mathrm{p}}^{2}}{2}-2 s\left(\gamma_{2}+2 \gamma_{4} \rho_{0}\right) \rho_{0}<0 .
$$

Here, we are predominantly interested in the normalGVD regime (where $s=-1$ ).

When both the cubic and quintic nonlinearity coefficients are positive $\left(\gamma_{2}>0\right.$ and $\left.\gamma_{4}>0\right)$, the $\mathrm{cw}$ solution is absolutely stable since condition (11b) can never be satisfied. For $\gamma_{2}>0$ and $\gamma_{4}<0$, MI appears when $\rho_{0}>\gamma_{2} / 2\left|\gamma_{4}\right|$. Analysis of the long-wavelength instability spectrum (the familiar bow-tie structure that is symmetric in $\Omega_{\mathrm{p}}$ ) shows that the most-unstable frequencies $\Omega_{\mathrm{p} 0}$ are obtained from $\Omega_{\mathrm{p} 0}^{2}=2\left(2\left|\gamma_{4}\right| \rho_{0}-\gamma_{2}\right) \rho_{0}$. For the opposite choice of signs $\left(\gamma_{2}<0\right.$ and $\left.\gamma_{4}>0\right)$, MI occurs for $\rho_{0}<\left|\gamma_{2}\right| / 2 \gamma_{4}$ and we have that $\Omega_{\mathrm{p} 0}^{2}=$ $2\left(\left|\gamma_{2}\right|-2 \gamma_{4} \rho_{0}\right) \rho_{0}$.

\section{B. Symmetry reduction}

To facilitate the integration of the quadrature equations, one expresses the desired solution phase as $\Psi(\tau, \zeta) \equiv \Psi_{\mathrm{d}}(\tau, \zeta)+K_{\mathrm{cw}} \zeta$, where $\Psi_{\mathrm{d}}(\tau, \zeta)$ describes the phase distribution across the soliton component while the cw phase (with $\Omega=0$ ) has been included explicitly at the outset. Substitution of the decomposition for $\Psi_{\mathrm{d}}$ into Eqs. (4a) and (4b) then yields

$$
\begin{gathered}
\frac{2}{\rho_{\mathrm{d}}}\left(\frac{\partial^{2} \rho_{\mathrm{d}}}{\partial \tau^{2}}-2 \kappa \frac{\partial^{2} \rho_{\mathrm{d}}}{\partial \zeta^{2}}\right)-\frac{1}{\rho_{\mathrm{d}}^{2}}\left[\left(\frac{\partial \rho_{\mathrm{d}}}{\partial \tau}\right)^{2}-2 \kappa\left(\frac{\partial \rho_{\mathrm{d}}}{\partial \zeta}\right)^{2}\right] \\
-4\left[\left(\frac{\partial \Psi_{\mathrm{d}}}{\partial \tau}\right)^{2}-2 \kappa\left(\frac{\partial \Psi_{\mathrm{d}}}{\partial \zeta}\right)^{2}\right] \\
+8\left(\alpha \frac{\partial \Psi_{\mathrm{d}}}{\partial \tau}+2 \kappa K_{\mathrm{cw}} \frac{\partial \Psi_{\mathrm{d}}}{\partial \zeta}\right) \\
+8\left[\left(\kappa K_{\mathrm{cw}}^{2}-\frac{1}{4 \kappa}\right)-\left(\gamma_{2}+\gamma_{4} \rho_{\mathrm{d}}\right) \rho_{\mathrm{d}}\right]=0,
\end{gathered}
$$

$$
\begin{aligned}
\rho_{\mathrm{d}} & \left(\frac{\partial^{2} \Psi_{\mathrm{d}}}{\partial \tau^{2}}-2 \kappa \frac{\partial^{2} \Psi_{\mathrm{d}}}{\partial \zeta^{2}}\right)+\left(\frac{\partial \Psi_{\mathrm{d}}}{\partial \tau} \frac{\partial \rho_{\mathrm{d}}}{\partial \tau}-2 \kappa \frac{\partial \Psi_{\mathrm{d}}}{\partial \zeta} \frac{\partial \rho_{\mathrm{d}}}{\partial \zeta}\right) \\
& -\left(\alpha \frac{\partial \rho_{\mathrm{d}}}{\partial \tau}+2 \kappa K_{\mathrm{cw}} \frac{\partial \rho_{\mathrm{d}}}{\partial \zeta}\right)=0
\end{aligned}
$$

One now introduces the lumped space-time variable $\xi$ from Eq. (5a), where the intrinsic velocity parameter is labelled as $V_{0 \mathrm{~d}}$ (the 'd' subscript refers to dark solitons). Equations (12a) and (12b) then reduce to

$$
\begin{aligned}
& \frac{d}{d \rho_{\mathrm{d}}}\left[\frac{1}{\rho_{\mathrm{d}}}\left(\frac{d \rho_{\mathrm{d}}}{d \xi}\right)^{2}\right]= 4\left(\frac{d \Psi_{\mathrm{d}}}{d \xi}\right)^{2} \\
&-8\left(\frac{\alpha-2 \kappa K_{\mathrm{cw}} V_{0 \mathrm{~d}}}{\sqrt{1-2 \kappa V_{0 \mathrm{~d}}^{2}}}\right) \frac{d \Psi_{\mathrm{d}}}{d \xi} \\
&-8\left[\beta_{\mathrm{cw}}-\left(\gamma_{2}+\gamma_{4} \rho_{\mathrm{d}}\right) \rho_{\mathrm{d}}\right]=0 \\
& \frac{d}{d \xi}\left[\left(\frac{d \Psi_{\mathrm{d}}}{d \xi}-\frac{\alpha-2 \kappa K_{\mathrm{cw}} V_{0 \mathrm{~d}}}{\sqrt{1-2 \kappa V_{0 \mathrm{~d}}^{2}}}\right) \rho_{\mathrm{d}}\right]=0
\end{aligned}
$$

where the cw dispersion relation for solutions with $\Omega=0$, namely $\kappa K_{\mathrm{cw}}^{2}-1 / 4 \kappa \equiv \beta_{\mathrm{cw}}$, has been introduced into Eq. (13a).

Direct integration of Eq. (13b) yields an ordinary differential equation for the soliton phase,

$$
\frac{d \Psi_{\mathrm{d}}}{d \xi}=\left(\frac{\alpha-2 \kappa K_{\mathrm{cw}} V_{0 \mathrm{~d}}}{\sqrt{1-2 \kappa V_{0 \mathrm{~d}}^{2}}}\right)+\frac{c_{1 \mathrm{~d}}}{\rho_{\mathrm{d}}},
$$

where $c_{1 \mathrm{~d}}$ is a constant of integration to be determined later (through an application of the solution boundary 
conditions). Substitution of Eq. (14a) into Eq. (13a) eliminates the phase gradient $d \Psi / d \xi$ yielding an ordinary differential equation for $\rho_{\mathrm{d}}$ :

$$
\begin{aligned}
\frac{d}{d \rho_{\mathrm{d}}}\left[\frac{1}{\rho_{\mathrm{d}}}\left(\frac{d \rho_{\mathrm{d}}}{d \xi}\right)^{2}\right]= & 4 \frac{c_{1 \mathrm{~d}}^{2}}{\rho_{\mathrm{d}}^{2}}-4\left(\frac{\alpha-2 \kappa K_{\mathrm{cw}} V_{0 \mathrm{~d}}}{\sqrt{1-2 \kappa V_{0 \mathrm{~d}}^{2}}}\right)^{2} \\
& -8\left[\beta_{\mathrm{cw}}-\left(\gamma_{2}+\gamma_{4} \rho_{\mathrm{d}}\right) \rho_{\mathrm{d}}\right] .
\end{aligned}
$$

System (13), comprising two coupled partial differential equations (in both space and time) has thus been reduced to system (14).

\section{Intensity quadrature}

The boundary conditions on the intensity quadrature are that $\rho_{\mathrm{d}} \rightarrow \rho_{0}$ and $\left(d \rho_{\mathrm{d}} / d \xi\right)^{2} \rightarrow 0$ as $\xi \rightarrow \pm \infty$, while $\rho_{\mathrm{d}} \rightarrow \rho_{1}$ and $\left(d \rho_{\mathrm{d}} / d \xi\right)^{2} \rightarrow 0$ as $\xi \rightarrow 0$ with $0<\rho_{1} \leq \rho_{0}$ for an intensity 'dip'. Direct integration of Eq. (14b) with respect to $\rho_{\mathrm{d}}$ leads to

$$
\begin{aligned}
\left(\frac{d \rho_{\mathrm{d}}}{d \xi}\right)^{2}= & \frac{8}{3} \gamma_{4} \rho_{\mathrm{d}}^{4}+4 \gamma_{2} \rho_{\mathrm{d}}^{3} \\
& -4\left[2 \beta_{\mathrm{cw}}+\left(\frac{\alpha-2 \kappa K_{\mathrm{cw}} V_{0 \mathrm{~d}}}{\sqrt{1-2 \kappa V_{0 \mathrm{~d}}^{2}}}\right)^{2}\right] \rho_{\mathrm{d}}^{2} \\
& +c_{2 \mathrm{~d}} \rho_{\mathrm{d}}-4 c_{1 \mathrm{~d}}^{2},
\end{aligned}
$$

where $c_{2 \mathrm{~d}}$ is a second constant to be determined. The derivation can be further facilitated by introducing a factorization to simplify the right-hand side of Eq. (15a). By respecting the solution asymptotics and recalling that $\left(d \rho_{\mathrm{d}} / d \xi\right)^{2}$ cannot be negative, we write

$$
\left(\frac{d \rho_{\mathrm{d}}}{d \xi}\right)^{2}=4\left(\rho_{0}-\rho_{\mathrm{d}}\right)^{2}\left(\rho_{\mathrm{d}}-\rho_{1}\right)\left(g_{1 \mathrm{~d}} \rho_{\mathrm{d}}+g_{0 \mathrm{~d}}\right),
$$

where $\rho_{0}$ is a double root, $\rho_{1}$ is a single root, and $g_{1 \mathrm{~d}}$ and $g_{0 \mathrm{~d}}$ are constants. Comparing Eqs. (15a) and (15b) leads to a system of five algebraic equations obtained by equating the powers of $\rho_{\mathrm{d}}$ :

$$
\begin{aligned}
g_{1 \mathrm{~d}} & \equiv \frac{2}{3} \gamma_{4}, \\
g_{0 \mathrm{~d}}-\rho_{1} g_{1 \mathrm{~d}}-2 g_{1 \mathrm{~d}} \rho_{0} & \equiv \gamma_{2}, \\
\rho_{0}^{2} g_{1 \mathrm{~d}}-\rho_{1} g_{0 \mathrm{~d}}-2 \rho_{0}\left(g_{0 \mathrm{~d}}-\rho_{1} g_{1 \mathrm{~d}}\right) & \equiv c_{3 \mathrm{~d}}, \\
4\left[2 \rho_{0} \rho_{1} g_{0 \mathrm{~d}}+\left(g_{0 \mathrm{~d}}-\rho_{1} g_{1 \mathrm{~d}}\right) \rho_{0}^{2}\right] & \equiv c_{2 \mathrm{~d}}, \\
\rho_{0}^{2} \rho_{1} g_{0 \mathrm{~d}} & \equiv c_{1 \mathrm{~d}}^{2},
\end{aligned}
$$

where we have introduced the lumped parameter

$$
c_{3 \mathrm{~d}} \equiv-\left[2 \beta_{\mathrm{cw}}+\left(\frac{\alpha-2 \kappa K_{\mathrm{cw}} V_{0 \mathrm{~d}}}{\sqrt{1-2 \kappa V_{0 \mathrm{~d}}^{2}}}\right)^{2}\right]
$$

for compactness. Solving Eqs. (15d)-(15f) leads to

$$
\begin{aligned}
& g_{0 \mathrm{~d}}=\gamma_{2}+2 \gamma_{4} \rho_{0}\left(1-\frac{A^{2}}{3}\right), \\
& c_{1 \mathrm{~d}}^{2}=\rho_{0}^{3}\left(1-A^{2}\right)\left[\gamma_{2}+2 \gamma_{4} \rho_{0}\left(1-\frac{A^{2}}{3}\right)\right], \\
& c_{2 \mathrm{~d}}=4 \rho_{0}^{2}\left[\gamma_{2}\left(3-2 A^{2}\right)+\frac{4}{3} \gamma_{4} \rho_{0}\left(2-A^{2}\right)^{2}\right],
\end{aligned}
$$

where we have introduced the notation $A^{2}+F^{2}=1$ and with $F^{2} \equiv \rho_{1} / \rho_{0}$ being the contrast parameter. Separating and integrating Eq. (15b), with $d \rho_{\mathrm{d}} / d \xi \geq 0$ in the domain $\xi \geq 0$, it can be shown that

$$
\rho_{\mathrm{d}}(\xi)=\rho_{0}-\frac{4 \beta_{\mathrm{d}}}{D \cosh \left(2 \sqrt{2 \beta_{\mathrm{d}}} \xi\right)+\left(\gamma_{2}+\frac{8}{3} \gamma_{4} \rho_{0}\right)},
$$

where

$$
\beta_{\mathrm{d}} \equiv \frac{\rho_{0} A^{2}}{2}\left[\gamma_{2}+\frac{2}{3} \gamma_{4} \rho_{0}\left(4-A^{2}\right)\right]
$$

and

$$
D \equiv \gamma_{2}+\frac{4}{3} \gamma_{4} \rho_{0}\left(2-A^{2}\right) .
$$

Note that the shape of the dark soliton pulse in Eqs. (17a) - (17c) is identical to that of its conventional counterpart [28], as must be the case (see Sec. III B).

\section{Intrinsic velocity}

To obtain an algebraic expression for the dark soliton intrinsic velocity $V_{0 \mathrm{~d}}$, we consider the asymptotic behaviour of the phase distribution in Eq. (14a). As $\xi \rightarrow \pm \infty$, one has that $\rho_{\mathrm{d}} \rightarrow \rho_{0}$ (the intensity of the solution approaches the cw background limit) and $d \Psi_{\mathrm{d}} / d \xi \rightarrow 0$. Hence, it follows that

$$
\frac{\alpha-2 \kappa K_{\mathrm{cw}} V_{0 \mathrm{~d}}}{\sqrt{1-2 \kappa V_{0 \mathrm{~d}}^{2}}}=-\frac{c_{1 \mathrm{~d}}}{\rho_{0}},
$$

where $c_{1 \mathrm{~d}}^{2}$ is related to solution parameters through Eq. (16b). One can then show that $V_{0 \mathrm{~d}}$ must satisfy the following general quadratic equation:

$$
\begin{aligned}
{\left[\left(2 \kappa K_{\mathrm{cw}}\right)^{2}+2 \kappa\left(\frac{c_{1 \mathrm{~d}}}{\rho_{0}}\right)^{2}\right] V_{0 \mathrm{~d}}^{2}-} & 2 \alpha\left(2 \kappa K_{\mathrm{cw}}\right) V_{0 \mathrm{~d}} \\
+ & +\alpha^{2}-\left(\frac{c_{1 \mathrm{~d}}}{\rho_{0}}\right)^{2}=0 .
\end{aligned}
$$

An identical equation for determining $V_{0 \mathrm{~d}}$ can be obtained from Eq. (15e). One must, of course, choose the root for $V_{0 \mathrm{~d}}$ that respects the signs in Eq. (18a). Combining with forward- and backward-propagating solutions, after some algebra it can be shown that 


$$
V_{0 \mathrm{~d}}= \pm \frac{\rho_{0}^{1 / 2} F \sqrt{\gamma_{2}+\frac{2}{3} \gamma_{4} \rho_{0}\left(2+F^{2}\right)}\left\{1+2 \kappa \rho_{0}\left[\left(2+F^{2}\right) \gamma_{2}+\frac{2}{3} \gamma_{4} \rho_{0}\left(F^{4}+2 F^{2}+3\right)\right]-2 \kappa \alpha^{2}\right\}^{1 / 2}+\alpha \sqrt{1+4 \kappa \beta_{\mathrm{cw}}}}{1+2 \kappa \rho_{0}\left[\left(2+F^{2}\right) \gamma_{2}+\frac{2}{3} \gamma_{4} \rho_{0}\left(F^{4}+2 F^{2}+3\right)\right]}
$$

(as with the bright solution, it will later prove convenient to release the \pm sign into the definition of $\xi$ to provide a more compact representation). Equation (19) combines into a single geometrical parameter the contribution from two distinct sources of motion: (i) the velocity relative to the laboratory frame due to the group speed (terms in $\alpha$ ), and (ii) the additional velocity change (relative to the black solution) due to finite grayness (terms in $F$ ).

Inspection of Eqs. (17a)-(17c) and (19) shows that a localized solution always exists for the purely-focusing nonlinearity with $\rho_{0}>0$ across the entire contrast range $0 \leq F^{2}<1$. For the competing nonlinearity $\gamma_{2}>0$ and $\gamma_{4}<0$, the solution requires $0<\rho_{0}<\rho_{0 \max }\left(F^{2}\right) \equiv$ $3 \rho_{0 \text { th }} /\left(3+F^{2}\right)$ (where $\rho_{0 \text { th }}=\gamma_{2} / 2\left|\gamma_{4}\right|$ is the cut-off intensity above which the $\mathrm{cw}$ background becomes modulationally unstable - see Sec. IV A). Similarly, the regime with $\gamma_{2}<0$ and $\gamma_{4}>0$ has $\rho_{0}>\rho_{0 \min }\left(F^{2}\right) \equiv 3 \rho_{0 \text { th }} /(2+$ $F^{2}$ ) (where $\rho_{0 \text { th }}=\left|\gamma_{2}\right| / 2 \gamma_{4}$ is the cut-off below which the $\mathrm{cw}$ background is unstable).

\section{E. Phase quadrature}

It now only remains to find an expression for the phase distribution. Combining Eqs. (14a) and (18a) leads to the quite general result

$$
\frac{d \Psi_{\mathrm{d}}}{d \xi}=\left(\frac{c_{1 \mathrm{~d}}}{\rho_{0}}\right)\left(\frac{\rho_{0}-\rho_{\mathrm{d}}}{\rho_{\mathrm{d}}}\right)
$$

which, after substituting for $\rho_{\mathrm{d}}(\xi)$ can be integrated exactly in closed form to yield

$$
\begin{aligned}
\Psi_{\mathrm{d}}(\xi)=\tan ^{-1}\{ & \left(\frac{A}{F}\right) \sqrt{\frac{\gamma_{2}+\frac{2}{3} \gamma_{4} \rho_{0}\left(2+F^{2}\right)}{\gamma_{2}+\frac{2}{3} \gamma_{4} \rho_{0}\left(3+F^{2}\right)}} \\
& \left.\times \tanh \left(\sqrt{2 \beta_{\mathrm{d}}} \xi\right)\right\} .
\end{aligned}
$$

As with the intensity quadrature, the phase distribution also possesses the same functional form as Herrmann's conventional dark soliton [28]. It is straightforward to show that the phase change across the pulse, defined as $\Delta \Psi_{\mathrm{d}} \equiv \Psi_{\mathrm{d}}(+\infty)-\Psi_{\mathrm{d}}(-\infty)$, is

$$
\Delta \Psi_{\mathrm{d}}=\pi-2 \tan ^{-1}\left\{\left(\frac{F}{A}\right) \sqrt{\frac{\gamma_{2}+\frac{2}{3} \gamma_{4} \rho_{0}\left(3+F^{2}\right)}{\gamma_{2}+\frac{2}{3} \gamma_{4} \rho_{0}\left(2+F^{2}\right)}}\right\}
$$

\section{MORE GENERAL SOLUTIONS}

\section{A. Frequency-velocity relations}

So far, we have considered only those solitary solutions that are centered on the carrier frequency (in the Fourier domain). However, by deploying the invariance laws detailed in Refs. [4-6], it is possible to find more general soliton families that are characterized by a finite frequency shift $\Omega$. Such a geometrical procedure naturally brings out a connection between $\Omega$ and the velocity $V$ parametrizing the coordinate transformation:

$$
\Omega(V) \equiv V \sqrt{\frac{1+4 \kappa \beta_{\mathrm{b}, \mathrm{cw}}}{1+2 s \kappa V^{2}}}+\alpha\left(\frac{1}{\sqrt{1+2 s \kappa V^{2}}}-1\right)
$$

where we select $s=+1$ for the bright solution and $s=-1$ for the dark.

After some algebra, it can be shown that $V$ must correspond to whichever branch of

$$
\begin{aligned}
& {\left[1+4 \kappa \beta_{\mathrm{b}, \mathrm{cw}}-2 s \kappa(\alpha+\Omega)^{2}\right] V^{2}} \\
& \quad+2 \alpha \sqrt{1+4 \kappa \beta_{\mathrm{b}, \mathrm{cw}}} V-2 \Omega\left(\alpha+\frac{1}{2} \Omega\right)=0
\end{aligned}
$$

vanishes when $\Omega=0$ (thereby ensuring that a non-zero $V$ can appear only in the presence of a non-zero $\Omega$ ). The frequency-shifted bright (see Fig. 1) and dark (see Fig. 2) solitons may then be stated as:

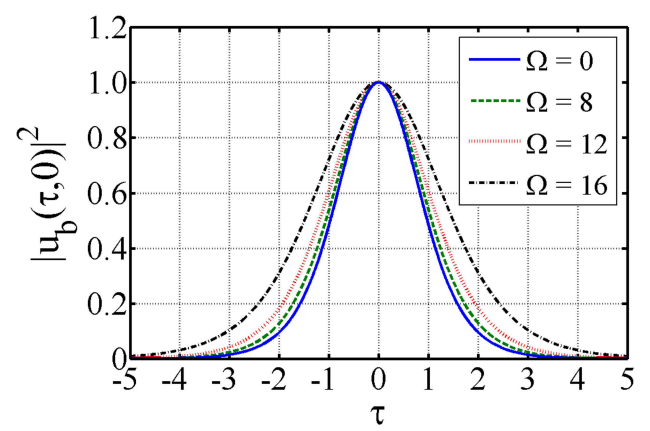

FIG. 1: (color online) Bright soliton intensity profiles [see solution (23a)] for increasing frequency shift $\Omega$ when the peak intensity is $\rho_{0}=1.0$. The pulse broadening effect (in essence, a Lorentz-like dilation in the presence of anomalous GVD [4]) is clearly visible. System parameters: $\gamma_{2}=+1$, $\gamma_{4}=-0.15, s=+1, \alpha=1.0$, and $\kappa=1.0 \times 10^{-3}$. Note that when plotting $\left|u_{\mathrm{b}}\right|^{2}$ as a function of $\xi$ [see solution (9)], the profiles are universal (that is, independent of $\kappa, \alpha$, and $V_{0 \mathrm{~b}}$ ) and there is no dilation effect. 


$$
u_{\mathrm{b}}(\tau, \zeta)=\left\{\frac{4 \beta_{\mathrm{b}}}{B \cosh \left[2 \sqrt{2 \beta_{\mathrm{b}}} \Theta_{\mathrm{b}}(\tau, \zeta)\right]+\gamma_{2}}\right\}^{1 / 2} \exp \left[i \Omega \tau \pm i \sqrt{1+4 \kappa \beta_{\mathrm{b}}-4 \kappa \Omega\left(\alpha+\frac{\Omega}{2}\right)} \frac{\zeta}{2 \kappa}\right] \exp \left(-i \frac{\zeta}{2 \kappa}\right)
$$

and

$$
\begin{aligned}
u_{\mathrm{d}}(\tau, \zeta)= & \left\{\rho_{0}-\frac{4 \beta_{\mathrm{d}}}{D \cosh \left[2 \sqrt{2 \beta_{\mathrm{d}}} \Theta_{\mathrm{d}}(\tau, \zeta)\right]+\left(\gamma_{2}+\frac{8}{3} \gamma_{4} \rho_{0}\right)}\right\}^{1 / 2} \\
& \times \exp \left[i \tan ^{-1}\left\{\left(\frac{A}{F}\right) \sqrt{\frac{\gamma_{2}+\frac{2}{3} \gamma_{4} \rho_{0}\left(2+F^{2}\right)}{\gamma_{2}+\frac{2}{3} \gamma_{4} \rho_{0}\left(3+F^{2}\right)}} \tanh \left[\sqrt{2 \beta_{\mathrm{d}}} \Theta_{\mathrm{d}}(\tau, \zeta)\right]\right\}\right] \\
& \times \exp \left[-i \Omega \tau \pm i \sqrt{1+4 \kappa \beta_{\mathrm{cw}}+4 \kappa \Omega\left(\alpha+\frac{\Omega}{2}\right)} \frac{\zeta}{2 \kappa}\right] \exp \left(-i \frac{\zeta}{2 \kappa}\right),
\end{aligned}
$$

respectively (see also Appendix C), where

$$
\begin{gathered}
\Theta_{\mathrm{b}, \mathrm{d}}(\tau, \zeta) \equiv \frac{\tau \mp W_{\mathrm{b}, \mathrm{d}} \zeta}{\sqrt{1+2 s \kappa W_{\mathrm{b}, \mathrm{d}}^{2}}} \\
W_{\mathrm{b}, \mathrm{d}}=\frac{V_{0 \mathrm{~b}, 0 \mathrm{~d}}+V_{\mathrm{b}, \mathrm{d}}}{1-2 s \kappa V_{0 \mathrm{~b}, 0 \mathrm{~d}} V_{\mathrm{b}, \mathrm{d}}}
\end{gathered}
$$

and

$$
V_{\mathrm{b}, \mathrm{d}}(\Omega)=\frac{(\Omega+\alpha) \sqrt{1+4 \kappa \beta_{\mathrm{b}, \mathrm{cw}}-4 s \kappa \Omega(\alpha+\Omega / 2)}-\alpha \sqrt{1+4 \kappa \beta_{\mathrm{b}, \mathrm{cw}}}}{1+4 \kappa \beta_{\mathrm{b}, \mathrm{cw}}-2 s \kappa(\Omega+\alpha)^{2}} .
$$

The parameter $V_{\mathrm{b}, \mathrm{d}}(\Omega)$, obtained from Eq. (22a), is analogous to the transverse velocity parameter from the theory of nonlinear beams $[34,35]$. For bright solitons, one can derive a compact expression for $W_{\mathrm{b}}$ such that

$$
W_{\mathrm{b}}=\frac{\alpha+\Omega}{\sqrt{1+4 \kappa \beta_{\mathrm{b}}-4 \kappa \Omega\left(\alpha+\frac{1}{2} \Omega\right)}} .
$$

We note that bright solitons are assigned a frequency shift such that $u_{\mathrm{b}} \propto \exp (i \Omega \tau)$ whereas dark solitons have $u_{\mathrm{d}} \propto \exp (-i \Omega \tau)$. Introducing such antisymmetry is somewhat arbitrary, but it allows the structure of Eqs. (22a) and (22b) to be preserved for both solution classes and that sign changes are most conveniently captured in the frequency-velocity relations solely by $s$ (rather than $s$ and $\Omega$ ).

Formally, one may recover the bright [5] and dark [6] spatiotemporal solitons of the cubically-nonlinear system by setting $\gamma_{2}=+1$ and $\left|\gamma_{4}\right| \rho_{0} \ll \mathcal{O}(1)$.

\section{B. Non-degenerate bistability}

By inspecting the solution continuum, one can search for parameter regimes where each wave class exhibits a non-degenerate bistability characteristic [27, 28]. This property is distinct from other types of bistable response, such as the familiar $\mathcal{S}$-shaped input-output curve of nonlinear cavities (present due to feedback modelled by ringresonator or Fabry-Pérot boundary conditions) [9] and from the case of degenerate solitons (where the integrated wave intensity can become a multi-valued function of the propagation constant if the derivative of the system nonlinearity functional satisfies certain constraints) [37].

Recalling that $\left|u_{\mathrm{b}}\right|^{2} \equiv \rho_{\mathrm{b}}$, for bright solitons (23a) the non-degenerate bistability condition $\rho_{\mathrm{b}}\left(\Theta_{\mathrm{b}}=\nu \Delta\right)=$ $\rho_{0} / 2[27]$ gives rise to the implicit equation

$$
\rho_{0}^{1 / 2}=\left(\frac{1}{2 \nu \Delta}\right) \frac{1}{\sqrt{\gamma_{2}+\frac{2}{3} \gamma_{4} \rho_{0}}} \cosh ^{-1}\left(\frac{3 \gamma_{2}+\frac{8}{3} \gamma_{4} \rho_{0}}{\gamma_{2}+\frac{4}{3} \gamma_{4} \rho_{0}}\right)
$$

where $2 \nu$ parametrizes the duration of the pulse (in its rest frame) in units of $\Delta \equiv \operatorname{sech}^{-1}\left(2^{-1 / 2}\right) \approx 0.8814$. For the competing nonlinearity with $\gamma_{2}>0$ and $\gamma_{4}<0$, there exist pairs of solitons that have the same full-widthat-half-maximum (FWHM) but different peak intensities (see Fig. 3). When $\left|\gamma_{4}\right| \rho_{0} \rightarrow 0$, the lower-branch solution in the $\left(\left|\gamma_{4}\right|, \rho_{0}\right)$ plane tends to $\rho_{0}=1 / \nu^{2} \gamma_{2}$ while the 


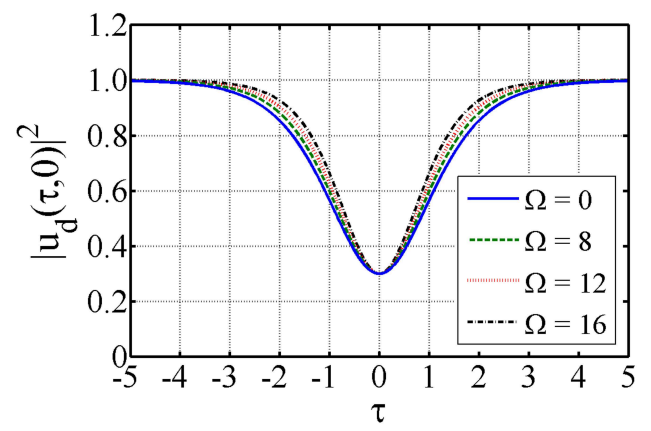

FIG. 2: (color online) Gray soliton intensity profiles [see solution (23b)] for increasing frequency shift $\Omega$ when $F=0.4$ and the cw intensity is $\rho_{0}=1.0$. Note the pulse narrowing (contraction) effect in the presence of normal GVD (in contrast to the contraction for anomalous GVD [4] - c.f.

Fig. 1). System parameters: $\gamma_{2}=+1, \gamma_{4}=-0.15, s=-1$, $\alpha=1.0$, and $\kappa=1.0 \times 10^{-3}$. Like its bright counterpart, $\rho_{\mathrm{d}}(\xi)[$ see $(17 \mathrm{a})]$ is universal so there is no dilation.

upper-branch diverges. Equation (24a) shows that other regimes for $\gamma_{2}$ and $\gamma_{4}$ tend to be monostable (i.e., there is no hysteresis in $\rho_{0}$ ).

Similarly, one can consider particular dark solitons in the continuum of solution (23b) that are prescribed by $\rho_{\mathrm{d}}\left(\Theta_{\mathrm{d}}=\nu \Delta\right)=\left(\rho_{0}+\rho_{1}\right) / 2[28]$, which corresponds to the condition

$$
\begin{aligned}
\rho_{0}^{1 / 2}= & \left(\frac{1}{2 \nu \Delta}\right)\left(\frac{1}{A}\right) \frac{1}{\sqrt{\gamma_{2}+\frac{2}{3} \gamma_{4} \rho_{0}\left(4-A^{2}\right)}} \\
& \times \cosh ^{-1}\left[\frac{3 \gamma_{2}+\frac{8}{3} \gamma_{4} \rho_{0}\left(3-A^{2}\right)}{\gamma_{2}+\frac{4}{3} \gamma_{4} \rho_{0}\left(2-A^{2}\right)}\right] .
\end{aligned}
$$

Pairs of non-degenerate bistable gray solutions sharing a common FWHM but with different $\mathrm{cw}$ intensities exist for the competing-nonlinearity $\gamma_{2}>0$ and $\gamma_{4}<0$. Analysis of Eq. (24b) shows that in the $\left(\left|\gamma_{4}\right|, \rho_{0}\right)$ plane, the lower-branch solution tends to $\rho_{0}=$

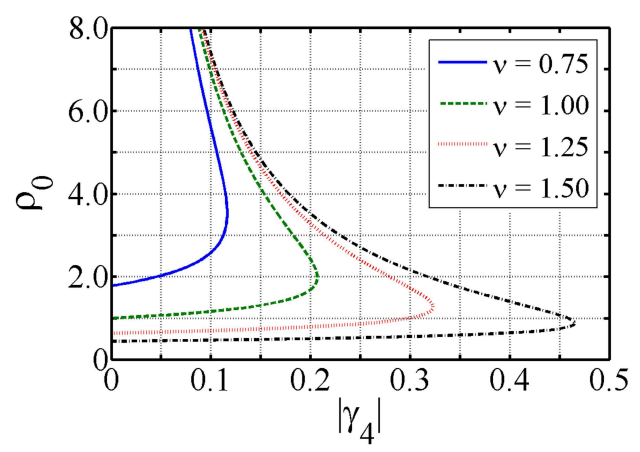

FIG. 3: (color online) Non-degenerate bistablity curves for bright solitons as predicted by Eq. (24a) for anomalous

GVD $(s=+1)$ in the competing-nonlinearity regime $\gamma_{2}=+1$ and $\gamma_{4}<0$ (other regimes tend to be monostable so that $\rho_{0}$ is a single-valued function).
$1 / \nu^{2} A^{2} \gamma_{2}$ while the upper-branch possesses a cut-off at point $\left(\left|\gamma_{4}\right|_{\text {crit }}, \rho_{0 \text { crit }}\right)$, where $\rho_{0 \text { crit }}=\left(4-A^{2}\right) / \gamma_{2}(\nu \Delta)^{2} A^{4}$ and $\left|\gamma_{4}\right|_{\text {crit }}=3\left(\gamma_{2} \nu \Delta\right)^{2} A^{4} / 2\left(4-A^{2}\right)^{2}$. Typical bistable curves are given in Fig. 4 for black and gray solitons.

\section{SLOWLY-VARYING ENVELOPES}

\section{A. Envelope equation}

The physical predictions of conventional pulse theory, viz. the parabolic envelope equation

$$
i\left(\frac{\partial u}{\partial \zeta}+\alpha \frac{\partial u}{\partial \tau}\right)+\frac{s}{2} \frac{\partial^{2} u}{\partial \tau^{2}}+\gamma_{2}|u|^{2} u+\gamma_{4}|u|^{4} u \simeq 0
$$

must emerge asymptotically from the spatiotemporal model in the limit of slowly-varying envelopes. The multi-faceted nature of that limit makes clear that stipulating $\kappa \simeq 0$ by itself is not a sufficient condition for the validity of Eq. (25a). Rather, one requires that all contributions from $\kappa \partial^{2} u / \partial \zeta^{2}$ must be negligible simultaneously when compared to those arising from the other terms in Eq. (2). One performs Taylor expansions on the exact solutions, all up to second-order smallness, so as the linear phase profile (which involves a ratio of small quantities) is handled correctly.

Under a Galilean boost to the local-time frame with coordinates $\tau_{\text {loc }} \equiv \tau-\alpha \zeta$ and $\zeta_{\text {loc }}=\zeta$, it is straightfor-
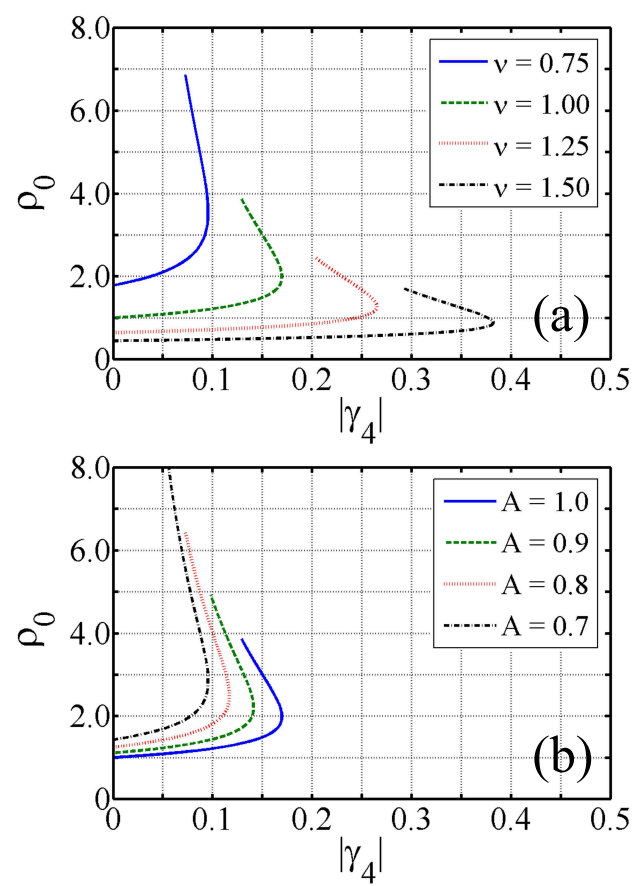

FIG. 4: (color online) Non-degenerate bistability curves for dark solitons [(a) black $(A=1)$ and (b) gray (with $\nu=1.0$ )

solutions] as predicted by Eq. (24b) for normal GVD

$(s=-1)$ in the competing-nonlinearity regime with $\gamma_{2}=+1$ and $\gamma_{4}<0$. 
ward to show that Eq. (25a) transforms into the standard cubic-quintic NLS-type model $[27,28]$,

$$
i \frac{\partial u}{\partial \zeta_{\text {loc }}}+\frac{s}{2} \frac{\partial^{2} u}{\partial \tau_{\text {loc }}^{2}}+\gamma_{2}|u|^{2} u+\gamma_{4}|u|^{4} u \simeq 0 .
$$

Equation (25b) thus describes pulses in a unique frame of reference (the one moving relative to the laboratory at the group velocity of pulses with slowly-varying envelopes in the $z$ direction).

\section{B. Intrinsic, transverse, and net velocities}

We begin by considering the behaviour of the various velocity parameters under the SVEA. For bright solitons, the limit $\kappa \beta_{\mathrm{b}} \ll \mathcal{O}(1)$ (corresponding to a near-negligible nonlinear phase shift) leads to $V_{0 \mathrm{~b}} \simeq \alpha \equiv V_{0 \mathrm{~b} \text { SVEA }}$. Applying the same limit yields a more involved result for dark solitons:

$$
V_{0 \mathrm{~d}} \simeq \rho_{0}^{1 / 2} F \sqrt{\gamma_{2}+\frac{2}{3} \gamma_{4} \rho_{0}\left(2+F^{2}\right)}+\alpha \equiv V_{0 \mathrm{~d} \text { SVEA }} .
$$

Both classes of solution thus have a contribution to the intrinsic velocity that is independent of frequency shift and (for dark solitons) grayness due to the fact that the pulses are always propagating with respect to the laboratory frame. The additional limit $|\kappa \Omega(\alpha+\Omega / 2)| \ll \mathcal{O}(1)$ (near-negligible frequency shift) gives transverse velocities $V_{\mathrm{b}, \mathrm{d}} \simeq \Omega \equiv V_{\mathrm{SVEA}}$ and, from Eq. (19), the net velocities become $W_{\mathrm{b}, \mathrm{d} \text { SVEA }} \simeq V_{0 \mathrm{~b}, 0 \mathrm{~d} \text { SVEA }}+V_{\mathrm{SVEA}}$.

For slowly-varying envelopes, one may now draw two conclusions about the properties of velocity parameters: (i) velocities combine additively (with Galilean-type rules) rather than geometrically (relativistic- or pseudorelativistic-type rules [4]), and (ii) transverse velocities and frequency shifts are interchangeable in the sense that they have the same mathematical status and are numerically equal to one another [a situation that is clearly distinct from the predictions of Eqs. (22a) and (23e)].

In the local-time frame, the term at $\alpha$ in $V_{0 \mathrm{~b}, 0 \mathrm{~d} \text { SVEA }}$ is transformed away and local velocities take on more familiar forms. On the one hand, bright solitons are characterized by $W_{\mathrm{bloc}}=V_{\mathrm{SVEA}}=\Omega$ so that pulses with $\Omega=0$ are strictly stationary in that frame. On the other hand, dark solitons have $W_{\text {d loc }}=V_{0 \mathrm{~d} \text { loc }}+V_{\mathrm{SVEA}}$, where $V_{0 \mathrm{dloc}}$ is defined to be the first term in Eq. (26). Black solutions (having $F=0=V_{0 \text { d loc }}$ ) with $\Omega=0$ thus have zero local net velocity and are also stationary.

It is now worth re-examining the linear boost described in the previous subsection. While introducing that coordinate change into the spatiotemporal model is always possible, it is problematic here for two principal reasons. Firstly, a mixed-derivative term must appear in the governing equation in order to retain an exact framework.
That is, Eq. (2) becomes

$$
\begin{aligned}
\kappa \frac{\partial^{2} u}{\partial \zeta_{\text {loc }}^{2}}+i \frac{\partial u}{\partial \zeta_{\text {loc }}}+\frac{1}{2}\left(s+2 \kappa \alpha^{2}\right) \frac{\partial^{2} u}{\partial \tau_{\text {loc }}^{2}} \\
\quad-2 \kappa \alpha \frac{\partial^{2} u}{\partial \zeta_{\text {loc }} \partial \tau_{\text {loc }}}+\gamma_{2}|u|^{2} u+\gamma_{4}|u|^{4} u=0
\end{aligned}
$$

and since 'preservation of exactness' is the central objective motivating our approach, simply ignoring or approximating the awkward fourth term is rather self-defeating. Secondly, and perhaps more importantly, the coordinates $\tau_{\text {loc }}$ and $\zeta_{\text {loc }}$ can no longer have quite the same significance now as they did previously because group velocities in the spatiotemporal formulation tend to have an inherent intensity dependence (a notable exception is the algebraic soliton discussed in Appendix A, which corresponds to the threshold for linear wave propagation [32]). That is, $1 / \alpha$ is strictly the (normalized) group velocity of a bright soliton with zero amplitude [c.f. Eq. (10) with $\Omega=0$ and $\kappa \beta_{\mathrm{b}}=0$ ] and of a black soliton on a zero-amplitude cw background [c.f. Eq. (19) with $\Omega=0$ and $\left.\kappa \beta_{\mathrm{cw}}=0\right]$. Hence, there can be no advantage (either physical or mathematical) in forcing the standard Galilean boost onto Eq. (2) and its solutions [though one can immediately write down the solitons of Eq. (27) directly from Eqs. (23a) - (23f)].

\section{Asymptotic solutions}

Solitons with slowly-varying envelopes in the laboratory and local-time frames [governed by Eqs. (25a) and (25b), respectively] can be obtained by applying the same limiting procedure to solutions (23a) and (23b). The asymptotic properties of velocity parameters are already known (see Sec. VIB), and in those same limits it follows that $\kappa W_{\mathrm{b}, \mathrm{d}}^{2} \ll \mathcal{O}(1)$. Hence, one has that $\Theta_{\mathrm{b}, \mathrm{d}}(\tau, \zeta) \simeq \tau \mp W_{\mathrm{b}, \mathrm{d}} \zeta$.

By expanding the propagation constants in a similar way, one can immediately write down the bright and dark solitons of Eq. (25a). Wavepackets propagating in the forward direction are well-behaved since all $\kappa$-dependent contributions vanish: the approximated solutions to the exact equation are exact solutions to the approximated equation. However, wavepackets travelling in the backward direction retain a rapid-phase factor $\exp (-i 2 \zeta / 2 \kappa)$ leading to the conclusion that Eq. (25a) has no analogue of backward spatiotemporal waves (being only parabolic rather than elliptic or hyperbolic, it supports propagation in a single longitudinal sense only [4]).

When considering the approximated forward solitons of Eq. (25a), one can boost to the local-time frame whereupon one recovers (generalizations of) known bright [27] and dark [28] solutions, 


$$
u_{\mathrm{b}}\left(\tau_{\text {loc }}, \zeta_{\text {loc }}\right) \simeq\left\{\frac{4 \beta_{\mathrm{b}}}{B \cosh \left[2 \sqrt{2 \beta_{\mathrm{b}}}\left(\tau_{\text {loc }}-\Omega \zeta_{\text {loc }}\right)\right]+\gamma_{2}}\right\}^{1 / 2} \exp \left[i \Omega \tau_{\text {loc }}+i\left(\beta_{\mathrm{b}}-\frac{\Omega^{2}}{2}\right) \zeta_{\text {loc }}\right]
$$

and

$$
\begin{aligned}
u_{\mathrm{d}}\left(\tau_{\text {loc }}, \zeta_{\text {loc }}\right) \simeq & \left\{\rho_{0}-\frac{4 \beta_{\mathrm{d}}}{D \cosh \left[2 \sqrt{2 \beta_{\mathrm{d}}}\left(\tau_{\text {loc }}-W_{\mathrm{d} \text { loc }} \zeta_{\text {loc }}\right)\right]+\left(\gamma_{2}+\frac{8}{3} \gamma_{4} \rho_{0}\right)}\right\}^{1 / 2} \\
& \times \exp \left[i \tan ^{-1}\left\{\left(\frac{A}{F}\right) \sqrt{\frac{\gamma_{2}+\frac{2}{3} \gamma_{4} \rho_{0}\left(2+F^{2}\right)}{\gamma_{2}+\frac{2}{3} \gamma_{4} \rho_{0}\left(3+F^{2}\right)}} \tanh \left[\sqrt{2 \beta_{\mathrm{d}}}\left(\tau_{\text {loc }}-W_{\mathrm{d} \text { loc }} \zeta_{\text {loc }}\right)\right]\right\}\right] \\
& \times \exp \left[-i \Omega \tau_{\text {loc }}+i\left(\beta_{\mathrm{cw}}+\frac{\Omega^{2}}{2}\right) \zeta_{\text {loc }}\right]
\end{aligned}
$$

where $W_{\mathrm{d} \text { loc }}=V_{0 \mathrm{~d} \text { loc }}+\Omega$ and

$$
V_{0 \mathrm{~d} \text { loc }} \equiv \rho_{0}^{1 / 2} F \sqrt{\gamma_{2}+\frac{2}{3} \gamma_{4} \rho_{0}\left(2+F^{2}\right)}
$$

are the local net and local intrinsic velocities, respectively. Wavepackets (28a) and (28b) satisfy Eq. (25b) exactly, reducing to their well-known cubic counterparts $[10,11]$ when $\gamma_{2}=+1$ and $\left|\gamma_{4}\right| \rho_{0} \ll \mathcal{O}(1)$.

\section{SOLITON STABILITY}

Finally, the behaviour of the new spatiotemporal solitons against perturbations to their local temporal shape is investigated through conventional stability criteria alongside supporting simulations. Numerical integration of Eq. (2) is facilitated through a generalization of the difference-differential algorithm [38] that accommodates the $i \alpha \partial / \partial \tau$ operator through fast Fourier transforms.

\section{A. Vakhitov-Kolokolov criterion}

The stability of localized excitation (28a) of Eq. (25b) has been discussed in detail by Gatz and Herrmann [27] within the context of the Vakhitov-Kolokolov (VK) integral criterion [39]. If $P$ is the pulse power defined by

$$
P \equiv \int_{-\infty}^{+\infty} d \tau_{\mathrm{loc}}\left|u_{\mathrm{b}}\right|^{2}
$$

then an arbitrary solution $u_{\mathrm{b}} \equiv u_{\mathrm{b}}\left(\tau_{\text {loc }}, \zeta_{\text {loc }}\right)$ is predicted to be stable against small perturbations provided that the derivative of $P$ satisfies the inequality

$$
\frac{d}{d \beta_{\mathrm{b}}} P\left(\beta_{\mathrm{b}}\right)>0
$$

where $\beta_{\mathrm{b}}$ is the propagation constant given by Eq. (8b). Physically-meaningful predictions from Eqs. (29a) and (29b) must be insensitive to frame-of-reference considerations since one evidently cannot have a wave that is both stable in the local-time frame and simultaneously unstable in any other frame [such as the laboratoryc.f. Eq. (25a)]. The $\kappa \partial^{2} / \partial \zeta^{2}$ operator in Eq. (2) tends to be predominantly geometrical in nature, and it typically introduces only a small correction to the solutions of Eq. (25a). We thus expect to find spatiotemporal solitons sharing very similar stability properties to their conventional counterparts, as demonstrated previously for the case of cubic systems [5].

Symmetry principles have also been deployed in the spatial domain to describe the stability characteristics of nonparaxial bright soliton beams beyond the cubic approximation using quasi-paraxial analyses [34, 40]. By recognizing that off-axis (Helmholtz-type) and on-axis (NLS-type) solutions are connected by a simple geometrical operation (a rotation of the observer's coordinate axes), it follows that oblique propagation effects can be eliminated for a single scalar beam with a careful choice of reference frame. One is then free to use established NLS-based methods [41, 42] for identifying frameindependent regions of stability in parameter space.

For the purely-positive nonlinearity, it is straightforward to show that solution (28a) has an integrated pulse intensity given by

$$
P\left(\beta_{\mathrm{b}}\right)=\sqrt{\frac{3}{2 \gamma_{4}}} \tan ^{-1}\left(\frac{1}{\gamma_{2}} \sqrt{\frac{16 \gamma_{4} \beta_{\mathrm{b}}}{3}}\right),
$$

which always has a positive gradient [43]. Analytic continuation allows one to immediately find the corresponding power in the competing-nonlinearity regime where $\gamma_{2}>0$ and $\gamma_{4}<0$ :

$$
P\left(\beta_{\mathrm{b}}\right)=\sqrt{\frac{3}{2\left|\gamma_{4}\right|}} \tanh ^{-1}\left(\frac{1}{\gamma_{2}} \sqrt{\frac{16\left|\gamma_{4}\right| \beta_{\mathrm{b}}}{3}}\right)
$$



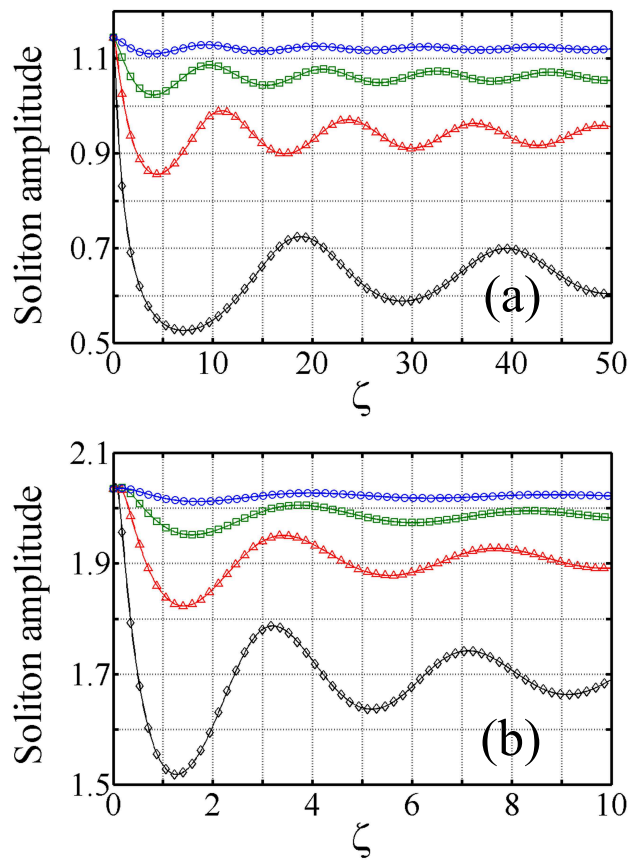

FIG. 5: (color online) Evolution of the bistable bright soliton peak amplitude when the initial waveform [as defined in Eq. (31)] resides on the (a) lower branch $\left(\rho_{0}=1.310\right)$ and

(b) upper branch $\left(\rho_{0}=4.141\right)$ - c.f. Fig. 3 with $\nu=1.0$. System parameters: $\gamma_{2}=+1, \gamma_{4}=-0.15, s=+1, \alpha=1.0$, $\kappa=1.0 \times 10^{-3}$. Blue circle: $\Omega=4$. Green square: $\Omega=8$.

Red triangle: $\Omega=12$. Black diamond: $\Omega=16$.

which also possesses a positive slope in the range $0 \leq$ $\beta_{\mathrm{b}}<3 \gamma_{2}^{2} / 16\left|\gamma_{4}\right|$ [43]. Solutions for the complementary regime $\gamma_{2}<0$ and $\gamma_{4}>0$ have

$$
P\left(\beta_{\mathrm{b}}\right)=\sqrt{\frac{3}{2 \gamma_{4}}}\left[\frac{\pi}{2}+\tan ^{-1}\left(\left|\gamma_{2}\right| \sqrt{\frac{3}{16 \gamma_{4} \beta_{\mathrm{b}}}}\right)\right],
$$

which tends to have a negative gradient in the allowed range of $\beta_{\mathrm{b}}$. Such waves are expected to be unstable according to the VK criterion [42], a prediction that has been confirmed numerically across a wide parameter space (we do not consider these solitons further).

\section{B. Perturbed bright solitons}

The numerical perturbative technique deployed here involves launching a pulse with the form

$$
u_{\mathrm{b}}(\tau, 0)=\left[\frac{4 \beta_{\mathrm{b}}}{B \cosh \left(2 \sqrt{2 \beta_{\mathrm{b}}} \tau\right)+\gamma_{2}}\right]^{1 / 2} \exp (i \Omega \tau),
$$

and observing propagation effects under the action of the system's internal dynamics. Initial data (31) corresponds to an exact soliton of Eq. (25a), or equivalently a spatiotemporal solution where the width factor $\left(1+2 \kappa W_{\mathrm{b}}^{2}\right)^{1 / 2}$ has been omitted. The frequency shift
$\Omega=4,8,12$, and 16 thus controls the strength of disturbance to the local temporal pulse shape.

We first consider a competing nonlinearity with $\gamma_{2}=$ +1 and $\gamma_{4}=-0.15$, which supports bistable solutions for $\nu=1.0$ with lower- and upper-branch peak intensities given by $\rho_{0} \approx 1.310$ and $\rho_{0} \approx 4.141$ (see Sec. VB). Simulations have demonstrated that evolution is generally adiabatic, with the pulse shape being maintained in $\zeta$. Parameters such as the peak amplitude (see Fig. 5), width, and area tend to undergo monotonically-decaying oscillations as the reshaping pulse evolves gradually towards a stationary state as $\zeta \rightarrow \infty$. A small amount of energy is shed in the form of radiation, and low-amplitude broad 'shoulders' can emerge at the base of the reshaping pulse in the presence of strong perturbations. The upper-branch solutions typically exhibit the same type of behaviour, except that the oscillations occur over a much shorter longitudinal scalelength and the early stages of propagation can involve an initial increase in the peak amplitude. If the radiation is regarded as a local loss mechanism (while the system remains globally conservative [5]), then the stationary states of Eq. (2) may be interpreted as attracting fixed points surrounded by wide basins of attraction [34].

For a purely positive nonlinearity, where $\gamma_{2}=+1$ and $\gamma_{4}=+0.15$, Eq. (24a) shows that there is a monostable solution with $\rho_{0} \approx 0.865$ when $\nu=1.0$. Simulations have revealed self-reshaping oscillations that are qualitatively similar to those encountered in the competing regime (compare the results in Fig. 6 to those in Fig. 5).

\section{Renormalized-momentum criterion}

The stability of conventional dark solitons in the localtime frame has previously been quantified by using an integral criterion that considers the renormalized momen-

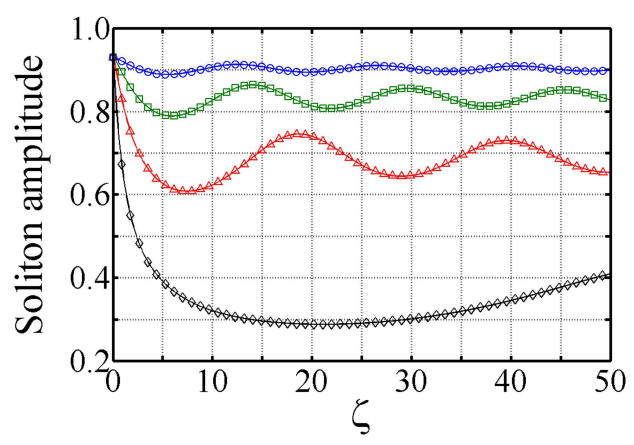

FIG. 6: (color online) Evolution of the peak amplitude when initial waveform (31) has $\rho_{0}=0.865$ and $\nu=1.0$. System parameters: $\gamma_{2}=+1, \gamma_{4}=-0.15, s=+1, \alpha=1.0$, $\kappa=1.0 \times 10^{-3}$. Blue circle: $\Omega=4$. Green square: $\Omega=8$.

Red triangle: $\Omega=12$. Black diamond: $\Omega=16$. 
tum $M_{\text {ren }}[44,45]$, where

$$
M_{\text {ren }} \equiv \frac{i}{2} \int_{-\infty}^{+\infty} d \tau_{\text {loc }}\left(u_{\mathrm{d}} \frac{\partial u_{\mathrm{d}}^{*}}{\partial \tau_{\mathrm{loc}}}-u_{\mathrm{d}}^{*} \frac{\partial u_{\mathrm{d}}}{\partial \tau_{\mathrm{loc}}}\right)\left(1-\frac{\rho_{0}}{\left|u_{\mathrm{d}}\right|^{2}}\right)
$$

Here, the formally-infinite contribution to the momentum integral from the cw background has been subtracted to leave a finite value, given by $M_{\text {ren }}$, that is associated with the localized excitation in $u_{\mathrm{d}}$. A dark solution to Eq. (25b) is then predicted to be stable against small disturbances if

$$
\frac{d}{d V_{\text {Od loc }}} s M_{\text {ren }}\left(V_{0 \mathrm{~d} \text { loc }}\right)>0
$$

is satisfied, where the derivative is taken with respect to the local intrinsic velocity parameter [given by Eq. (28c)]. Pelinovsky et al. [45] have applied the renormalizedmomentum approach (in parallel with asymptotic methods and numerical analyses) to study the stability properties of conventional dark solitons in the presence of competing, saturable, and transiting nonlinearities. As in the case of bright pulses, such predictions must be frameindependent if they are to be truly meaningful and hence one expects Eqs. (32a) and (32b) play a key role in quantifying dark pulses in spatiotemporal contexts [6].

Extensive simulations have shown that the exact dark solitons of Eq. (2) tend to propagate with invariant profile, and they demonstrate robustness as predicted by Eqs. (32a) and (32b).

\section{Perturbed dark solitons}

To test the stability of spatiotemporal dark solitons, we launch pulses of the form given by Eq. (23b) but where the factor $\left(1-2 \kappa W_{\mathrm{d}}^{2}\right)^{1 / 2}$ is omitted from $\Theta_{\mathrm{d}}(\tau, \zeta)$ :

$$
\begin{aligned}
u_{\mathrm{d}}(\tau, 0)= & \left\{\rho_{0}-\frac{4 \beta_{\mathrm{d}}}{D \cosh \left(2 \sqrt{2 \beta_{\mathrm{d}}} \tau\right)+\left(\gamma_{2}+\frac{8}{3} \gamma_{4} \rho_{0}\right)}\right\}^{1 / 2} \\
& \times \exp \left[i \operatorname { t a n } ^ { - 1 } \left\{\left(\frac{A}{F}\right) \sqrt{\frac{\gamma_{2}+\frac{2}{3} \gamma_{4} \rho_{0}\left(2+F^{2}\right)}{\gamma_{2}+\frac{2}{3} \gamma_{4} \rho_{0}\left(3+F^{2}\right)}}\right.\right. \\
& \left.\left.\times \tanh \left(\sqrt{2 \beta_{\mathrm{d}}}\right)\right\}\right] \\
& \times \exp (-i \Omega \tau) .
\end{aligned}
$$

Such an input wave corresponds to an exact solution of Eq. (25a). To accommodate the linear phase ramp associated with finite- $\Omega$ considerations, simulations are performed in a frame of reference wherein the factor $\exp (-i \Omega \tau)$ is eliminated. Results from simulations are then transformed back to $(\tau, \zeta)$ coordinates. We begin by considering perturbed black solitons (where $A=1$ ). For consistency, parameters $\gamma_{2}=+1$ and $\gamma_{4}=-0.15$ are retained in which case Eq. (24b) shows there are two
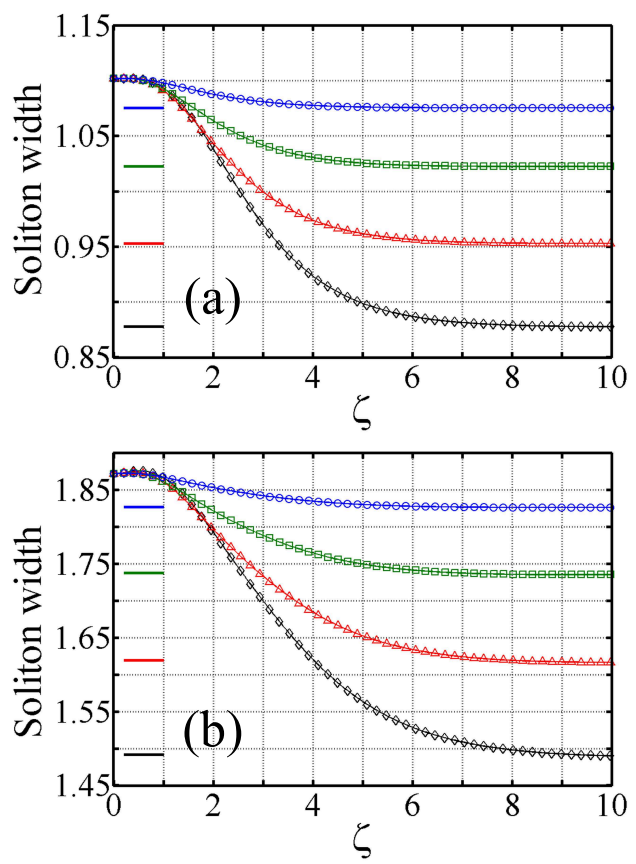

FIG. 7: (color online) Evolution of the bistable black soliton full width when the initial waveform [as defined in Eq. (33)] resides on the (a) lower branch $\left(\rho_{0}=1.4866\right)$ and (b) upper branch $\left(\rho_{0}=3.0183\right)$ - c.f. Fig. 4 (horizontal bars indicate theoretical predictions). System parameters: $\gamma_{2}=+1, \gamma_{4}=-0.15, s=-1, \alpha=1.0, \kappa=1.0 \times 10^{-3}$. Blue circle: $\Omega=4$. Green square: $\Omega=8$. Red triangle: $\Omega=12$. Black diamond: $\Omega=16$.

solutions for $\nu=1$ with $\mathrm{cw}$ intensities $\rho_{0} \simeq 1.486$ and $\rho_{0} \simeq 3.018$ (see Fig. 4). The temporal full-width of the initial waveform, denoted by $w_{0} \equiv\left(2 \beta_{\mathrm{d}}\right)^{-1 / 2}$, is broader than that for the exact solution. Numerical analyses demonstrate that as $\zeta \rightarrow \infty$, the reshaping pulse sheds radiation in the form of low-amplitude ripples across the cw background (an effect that becomes slightly more pronounced with increasing $\Omega$ ). The localized component otherwise tends to evolve adiabatically towards a stationary state, preserving its blackness and with a general shape prescribed by solution (23b). The pulse width can be seen to decrease smoothly towards the asymptotic value $w_{\infty}=w_{0}\left(1-2 \kappa W_{\mathrm{d}}^{2}\right)^{1 / 2}$ (see Fig. 7).

Gray solitons perturbed in the same way share similar stability properties to those of their black counterparts. One key distinction is that, for $F \neq 0$, the grayness of the solution is not quite preserved as $\zeta \rightarrow \infty$. These small changes in $F$, embodied by $F \rightarrow F(\zeta)$, are connected to variations in $V_{0 \mathrm{~d}}(F)$ so that the evolving waveform is subject to a slight drift instability (though the trajectory is still predominantly linear). That is, the center of the Gray pulse travels (approximately) along the characteristic $\tau-W_{\mathrm{d}}(\zeta) \zeta=$ const., where the functional form of $V_{0 \mathrm{~d}}(F)$ is preserved [c.f. Eq. (19)] but $F(\zeta)$ must be computed from the numerical solution (illustrative results are shown in Fig. 8). As $\zeta \rightarrow \infty$, stationary states tend to 


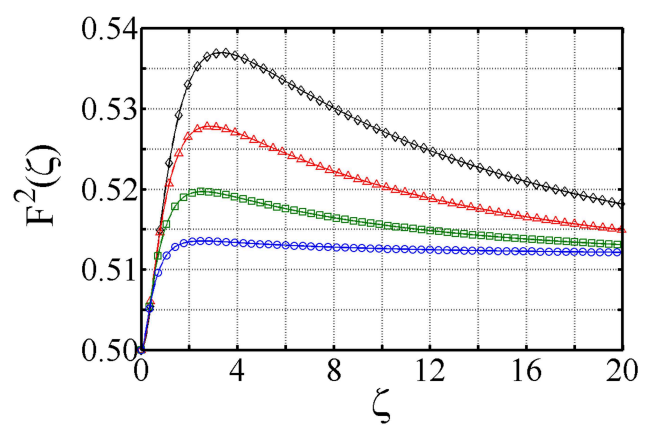

FIG. 8: (color online) Evolution of the gray soliton contrast parameter when the initial waveform [as defined in Eq. (33)] has $\rho_{0}=2.0$ and $F^{2}(0)=0.5$. System parameters: $\gamma_{2}=+1, \gamma_{4}=-0.15, s=-1, \alpha=1.0, \kappa=1.0 \times 10^{-3}$. Blue circle: $\Omega=4$. Green square: $\Omega=8$. Red triangle: $\Omega=12$. Black diamond: $\Omega=16$.

emerge with $F$ values that are slightly greater than the initial value.

\section{CONCLUSIONS}

We have considered in some detail a spatiotemporal scalar wave equation with cubic-quintic nonlinearity, deploying a combination of methods (direct integration and coordinate transformations) to derive exact analytical bright and dark solitons. These new classes of wavepacket are localized in the time domain, and comprise distinct solution branches describing propagation in the forward and backward longitudinal directions relative to the laboratory frame of reference. We have rigorously proved that in the limit of slowly-varying envelopes and after transformation to the local-time frame, bright [27] and dark solitons [28] of the NLS-type model emerge asymptotically from the forward-travelling spatiotemporal solutions. We also recover the corresponding solitons of the cubic system $[5,6]$ in the limit of a negligible quintic response. Established analytical methods $[42,45]$ have been used to assess the stability properties of the new solitons, with results from simulations fully supporting theoretical predictions. The spatiotemporal solutions reported here have generally been found to behave as robust attractors that tend to be highly stable against perturbations to the local temporal pulse profile.

To date, we have considered exact bright and dark spatiotemporal solitons for cubic $[5,6]$, cubic-quintic, and saturable - see companion article [46]-dispersive systems. Together these simple nonlinearity models have played an important role in developing our understanding of wave physics and envelope propagation, largely because in each case the governing equations can be solved analytically. There remains another fundamental solution class of particular interest in cubic-quintic systems, namely that of antidark solitons [47]. We have recently discovered that Eq. (2) supports such excitations that deserve careful attention.

Our latest research is concerned with more general dispersive nonlinearities, identifying connections between spatiotemporal envelope models similar to Eq. (2) and their (real) Klein-Gordon counterparts. Deducing a mapping between these two universal types of governing equation is potentially useful as it provides a platform for the direct interchange of solitary solutions between, for example, the fields of optics and particle physics. It would also be fascinating to extend our spatiotemporal considerations beyond the standard solitary structures (bright, dark, boundary, and antidark waves), e.g., to seek generalizations of the Peregrine soliton [48] and develop relativistic- and pseudorelativistic-type formulations of rogue- $[49,50]$ and shock-wave [51] phenomena.

\section{Appendix A: Algebraic solitons}

\section{Exact solutions \& asymptotics}

A class of weakly-localized nonlinear wave can be obtained from solution (23a) in the competing-nonlinearity regime $\gamma_{2}<0$ and $\gamma_{4}>0$. Spatiotemporal algebraic solitons correspond to the case of a vanishing propagation constant (obtained by setting $\beta_{\mathrm{b}} \rightarrow 0$ ), and for the cubic-quintic system possess much slower Lorentzian (rather than exponential) asymptotics [32, 42]. By considering binomial expansions to leading-order in $\beta_{\mathrm{b}}$, namely $\cosh \left(2 \sqrt{2 \beta_{\mathrm{b}}} \Theta_{\mathrm{b}}\right) \simeq 1+\left(4 \beta_{\mathrm{b}}\right) \Theta_{\mathrm{b}}^{2}$ and $B \simeq$ $\left|\gamma_{2}\right|\left[1+\left(2 \gamma_{4} / 3 \gamma_{2}^{2}\right)\left(4 \beta_{\mathrm{b}}\right)\right]$, it can be shown that there exists finite-amplitude forward- and backward-propagating wavepackets

$$
\begin{aligned}
u_{\mathrm{a}}(\tau, \zeta)= & \sqrt{\frac{3\left|\gamma_{2}\right|}{2 \gamma_{4}}}\left[\left(\frac{3 \gamma_{2}^{2}}{2 \gamma_{4}}\right) \Theta_{\mathrm{a}}^{2}(\tau, \zeta)+1\right]^{-1 / 2} \\
& \times \exp \left[i \Omega \tau \pm i \sqrt{1-4 \kappa \Omega\left(\alpha+\frac{\Omega}{2}\right)} \frac{\zeta}{2 \kappa}\right] \\
& \times \exp \left(-i \frac{\zeta}{2 \kappa}\right)
\end{aligned}
$$

where

$$
\Theta_{\mathrm{a}}(\tau, \zeta) \equiv \frac{\tau \mp W_{\mathrm{a}} \zeta}{\sqrt{1+2 \kappa W_{\mathrm{a}}^{2}}}
$$

(the 'a' subscript denotes algebraic solitons), and the net velocity parameter $W_{\mathrm{a}}$ is identical to $W_{\mathrm{b}}$ in Eq. (23f) but with the factor $4 \kappa \beta_{\mathrm{b}}$ omitted. The intensity of these Lorentzian-shaped solutions falls off according to an inverse-square law, $\sim 1 / \Theta_{\mathrm{a}}^{2}$, so the tails are relatively broad (it is in this sense that algebraic excitations are weakly localized-see Fig. 9).

By taking the forward-propagating algebraic soliton and applying the multiple-limit procedure as described in Sec. VI, one can subsequently transform to the localtime frame and hence find the corresponding solution of 


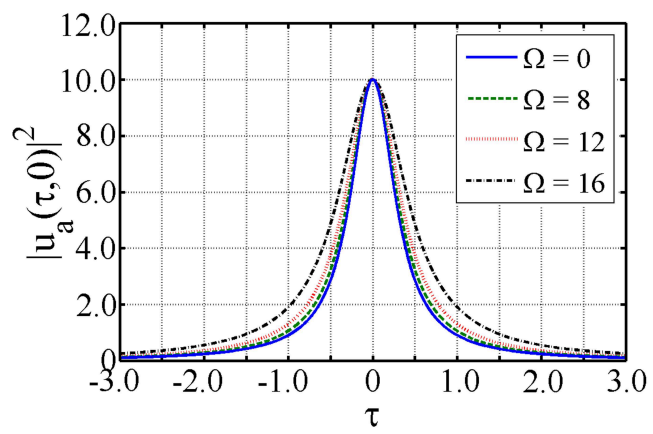

FIG. 9: (color online) Algebraic soliton intensity profile according to solution (A1a). The tails of the distribution are

Lorentzian, falling off like $1 / \tau^{2}$ as $\tau \rightarrow \pm \infty$, while anomalous GVD leads to a broadening of the pulse width. System parameters: $\gamma_{2}=-1, \gamma_{4}=+0.15, s=+1, \alpha=1.0$,

$$
\kappa=1.0 \times 10^{-3} \text {. }
$$

Eq. (25b):

$$
\begin{aligned}
u_{\mathrm{a}}\left(\tau_{\text {loc }}, \zeta_{\text {loc }}\right) \simeq & \sqrt{\frac{3\left|\gamma_{2}\right|}{2 \gamma_{4}}}\left[\left(\frac{3 \gamma_{2}^{2}}{2 \gamma_{4}}\right)\left(\tau_{\text {loc }}-\Omega \zeta_{\text {loc }}\right)^{2}+1\right]^{-1 / 2} \\
& \times \exp \left(i \Omega \tau_{\text {loc }}-i \frac{\Omega^{2}}{2} \zeta_{\text {loc }}\right) .
\end{aligned}
$$

From Eq. (30c), it is easy to see that the integrated power in solution (A2) remains finite and assumes the value of $P(0)=\left(3 / 2 \gamma_{4}\right)^{1 / 2} \pi$. The absence of any free internal parameter (such as $\beta_{\mathrm{b}}$ ) has implications for the algebraic soliton stability problem.

\section{Instability of algebraic solitons}

Since the integrated pulse power of the hyperbolic soliton with $\gamma_{2}<0$ and $\gamma_{4}>0$ retains a negative gradient $d P_{\mathrm{b}} / d \beta_{\mathrm{b}}$ as $\beta_{\mathrm{b}} \rightarrow 0$ [see Eq. (30c)], one can infer that

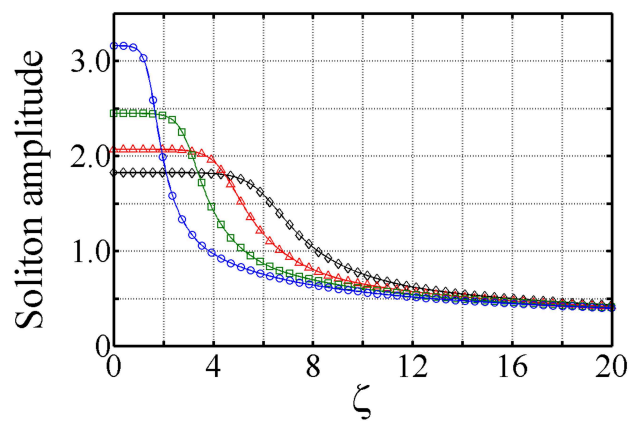

FIG. 10: (color online) Instability of spatiotemporal algebraic solitons. Initial conditions correspond to exact solution (A1a), and where the peak intensity is $\rho_{0}=3\left|\gamma_{2}\right| / 4 \gamma_{4}$. System parameters: $\gamma_{2}=-1, s=+1$, $\alpha=1.0, \kappa=1.0 \times 10^{-3}$. Blue circle: $\gamma_{4}=0.15$. Green square: $\gamma_{4}=0.25$. Red triangle: $\gamma_{4}=0.35$. Black diamond:

$$
\gamma_{4}=0.45 \text {. }
$$

algebraic solution (A2) must be always unstable since it does not satisfy the VK inequality of Eqs. (29a) and (29b). Analysis of these conventional weakly-localized states (in terms of both multiple-scale perturbation theory and supporting simulations) $[32,42]$ connects that instability to resonant interactions with infinitely-long linear waves.

Numerical integration of Eq. (2) with exact solutions (A1a)-(A1b) as initial conditions has provided compelling evidence that such instability persists in the spatiotemporal regime. Typical evolution of an algebraic soliton is shown in Fig. 10 for $\gamma_{2}=-1$ and $\gamma_{4}=+0.15$. The weakly-localized state survives largely intact for a short distance in $\zeta$ before starting to transform into a plateau-type structure accompanied by the emission of a ripple-type radiation pattern. Increasing $\gamma_{4}$ reduces the peak intensity of the initial algebraic soliton, and delays (but does not suppress) the onset of a qualitatively similar dispersive-broadening instability (see Fig. 9).

\section{Appendix B: Boundary solitons}

\section{Exact solutions \& asymptotics}

Equation (2) supports a class of partially delocalized wave in the form of a spatiotemporal kink or boundary soliton for anomalous $\operatorname{GVD}(s=+1)$ and the competing-nonlinearity regime $\gamma_{2}>0$ and $\gamma_{4}<0$. Such solutions connect (modulationally stable) plateau regions of zero amplitude to regions of constant amplitude $\left(3 \gamma_{2} / 4\left|\gamma_{4}\right|\right)^{1 / 2}$. Boundary solitons are thus amplitudetopological excitations rather than phase-topological (since there is no phase change across the temporal extent of the wave), and are given by

$$
\begin{aligned}
& u_{\mathrm{k} \pm}(\tau, \zeta)= \\
& \sqrt{\frac{4 \beta_{\mathrm{k}}}{\gamma_{2}}}\left\{\exp \left[ \pm 2 \sqrt{2 \beta_{\mathrm{k}}} \Theta_{\mathrm{k}}(\tau, \zeta)\right]+1\right\}^{-1 / 2} \\
& \quad \times \exp \left[i \Omega \tau \pm i \sqrt{\left.1+4 \kappa \beta_{\mathrm{k}}-4 \kappa \Omega\left(\alpha+\frac{\Omega}{2}\right) \frac{\zeta}{2 \kappa}\right]}\right. \\
& \quad \times \exp \left(-i \frac{\zeta}{2 \kappa}\right),
\end{aligned}
$$

where

$$
\Theta_{\mathrm{k}}(\tau, \zeta) \equiv \frac{\tau \mp W_{\mathrm{k}} \zeta}{\sqrt{1+2 \kappa W_{\mathrm{k}}^{2}}}
$$

and the net velocity parameter $W_{\mathrm{k}}$ is identical in form to $W_{\mathrm{b}}$ given in Eq. (23f) but with $\beta_{\mathrm{b}}$ replaced by $\beta_{\mathrm{k}} \equiv$ $3 \gamma_{2}^{2} / 16\left|\gamma_{4}\right|$. The \pm sign in the argument of the realexponential function (which can be selected independently of the sign flagging the propagation direction in the complex-exponential function) determines the parity of the wave [classified as kink $(-)$ or antikink $(+)$ ], where $\left|u_{\mathrm{k} \pm}\right| \rightarrow 0$ as $\Theta_{\mathrm{k}} \rightarrow \pm \infty$ (see Fig. 11). 


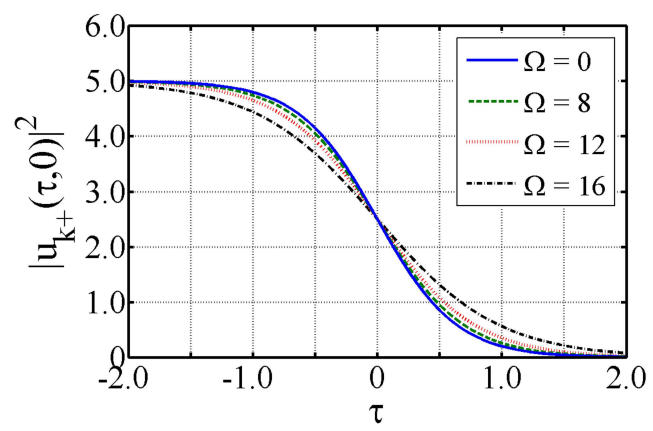

FIG. 11: (color online) Boundary (antikink) soliton intensity profile according to solution (B1a), which plateaus towards the constant value $4 \beta_{\mathrm{k}} / \gamma_{2}$ as $\tau \rightarrow-\infty$ and falls off exponentially toward zero as $\tau \rightarrow+\infty$. Anomalous GVD also leads to a broadening of the transition region (taken to be a measure of the pulse duration). System parameters: $\gamma_{2}=+1, \gamma_{4}=-0.15, s=+1, \alpha=1.0, \kappa=1.0 \times 10^{-3}$.

By taking the forward-propagating boundary soliton and applying the multiple-limit procedure as described in Sec. VI, one can subsequently transform to the localtime frame and hence find the corresponding solution of Eq. (25b) first proposed by Gagnon [29]:

$$
\begin{aligned}
& u_{\mathrm{k} \pm}\left(\tau_{\text {loc }}, \zeta_{\text {loc }}\right) \simeq \\
& \quad \sqrt{\frac{4 \beta_{\mathrm{k}}}{\gamma_{2}}}\left\{\exp \left[ \pm 2 \sqrt{2 \beta_{\mathrm{k}}}\left(\tau_{\text {loc }}-\Omega \zeta_{\text {loc }}\right)\right]+1\right\}^{-1 / 2} \\
& \quad \times \exp \left[i \Omega \tau_{\text {loc }}+i\left(\beta_{\mathrm{k}}-\frac{\Omega^{2}}{2}\right) \zeta_{\text {loc }}\right] .
\end{aligned}
$$

Computational studies by Kim and Moon [31] have previously found that these wavepackets are typically very robust entities that tend to be resilient even to strong perturbations (such as collisions with bright solitons).

\section{Perturbed boundary solitons}

In the spatial domain, amplitude kinks of a generalized cubic-quintic Helmholtz equation have been reported and their stability demonstrated numerically [40]. Using the same symmetry principles discussed in Sec. VII, one would expect the corresponding solutions in the time domain [that is, Eq. (B1a)] to demonstrate a similar degree of robustness.

For completeness, simulations with Eq. (2) are now used to test boundary soliton stability against local (temporal) shape fluctuations. We consider the antikink initial-value problem defined by

$$
u_{\mathrm{k}+}(\tau, 0)=\sqrt{\frac{4 \beta_{\mathrm{k}}}{\gamma_{2}}}\left[\exp \left(2 \sqrt{2 \beta_{\mathrm{k}}} \tau\right)+1\right]^{-1 / 2} \exp (i \Omega \tau),
$$

which corresponds to a perturbed solution of Eq. (2) but satisfies Eq. (25a) exactly [the geometry of initial data (B3) is thus equivalent to that used throughout the preceding computations]. As in the case of dark solitons, numerical calculations are most conveniently performed in a frame of reference where the linear phase ramp from the $\exp (i \Omega \tau)$ factor is eliminated and datasets are transformed back to the $(\tau, \zeta)$ frame when necessary.

The characteristic width of the initial condition (quantifying the size of the transition region between the zero- and finite-amplitude domains) is defined as $w_{0} \equiv$ $\left(2 \beta_{\mathrm{k}}\right)^{-1 / 2}$. Since $w_{0}$ is less than that needed for the exact spatiotemporal solution, we expect the waveform to transform smoothly into a stationary state of Eq. (2) whose asymptotic width is predicted to be $w_{\infty}=w_{0}(1+$ $\left.2 \kappa W_{\mathrm{k}}^{2}\right)^{1 / 2}$. The evolution is predominantly adiabatic (see Fig. 12) aside from a small-amplitude radiation ripple pattern that tends to develop on top of the high-intensity portion of the solution.

\section{Appendix C: Alternative representations}

Solitons of the cubic nonlinearity are perhaps the best known [10], where the fundamental bright and dark solutions are expressed in terms of hyperbolics sech and tanh, respectively. It is thus instructive to couch the cubic-quintic solutions in terms of these same functions.

To that end, one can show that bright soliton (23a) has an alternative representation that involves a combination of sech functions:

$$
\begin{aligned}
u_{\mathrm{b}}(\tau, \zeta)= & \left(2 \beta_{\mathrm{b}}\right)^{1 / 2} \\
& \times \frac{\operatorname{sech}\left[\sqrt{2 \beta_{\mathrm{b}}} \Theta_{\mathrm{b}}(\tau, \zeta)\right]}{\sqrt{\gamma_{2}+\frac{4}{3} \gamma_{4} \rho_{0}-\frac{2}{3} \gamma_{4} \rho_{0} \operatorname{sech}^{2}\left[\sqrt{2 \beta_{\mathrm{b}} \Theta_{\mathrm{b}}(\tau, \zeta)}\right]}} \\
& \times \exp (i \Omega \tau) \\
& \times \exp \left[ \pm i \sqrt{1+4 \kappa \beta_{\mathrm{b}}-4 \kappa \Omega\left(\alpha+\frac{\Omega}{2}\right)} \frac{\zeta}{2 \kappa}\right] \\
& \times \exp \left(-i \frac{\zeta}{2 \kappa}\right), \quad(\mathrm{C} 1)
\end{aligned}
$$

where the cubic solution [5],

$$
u_{\mathrm{b}}(\tau, \zeta) \propto \rho_{0}^{1 / 2} \operatorname{sech}\left[\left(\gamma_{2} \rho_{0}\right)^{1 / 2} \Theta_{\mathrm{b}}(\tau, \zeta)\right],
$$

is an obvious limit when $\left|\gamma_{4}\right| \rho_{0} /\left|\gamma_{2}\right| \ll \mathcal{O}(1)$ and $\gamma_{2}>$ 0 . Similarly, dark soliton (23b) can be described by a solution where the intensity-phase contribution appears as a complex number in Cartesian form,

$$
\rho_{\mathrm{d}}^{1 / 2}(\tau, \zeta) \exp \left[i \Psi_{\mathrm{d}}(\tau, \zeta)\right] \equiv \mathcal{R}(\tau, \zeta)+i \mathcal{I}(\tau, \zeta),
$$

where $\mathcal{R}$ and $\mathcal{I}$ are real functions to be determined. Since $\rho_{\mathrm{d}}=\mathcal{R}^{2}+\mathcal{I}^{2}$ and $\tan \Psi_{\mathrm{d}}=\mathcal{I} / \mathcal{R}$, it follows that 


$$
\begin{aligned}
u_{\mathrm{d}}(\tau, \zeta)= & \rho_{0}^{1 / 2} \frac{\left[\gamma_{2}+\frac{2}{3} \gamma_{4} \rho_{0}\left(3-A^{2}\right)\right]^{1 / 2} A \tanh \left[\sqrt{2 \beta_{\mathrm{d}}} \Theta_{\mathrm{d}}(\tau, \zeta)\right]-i\left[\gamma_{2}+\frac{2}{3} \gamma_{4} \rho_{0}\left(4-A^{2}\right)\right]^{1 / 2} F}{\sqrt{\gamma_{2}+\frac{2}{3} \gamma_{4} \rho_{0}\left(4-A^{2}\right)-\frac{2}{3} \gamma_{4} \rho_{0} A^{2} \tanh ^{2}\left[\sqrt{2 \beta_{\mathrm{d}}} \Theta_{\mathrm{d}}(\tau, \zeta)\right]}} \\
& \times \exp \left[-i \Omega \tau \pm i \sqrt{1+4 \kappa \beta_{\mathrm{cw}}+4 \kappa \Omega\left(\alpha+\frac{\Omega}{2}\right)} \frac{\zeta}{2 \kappa}\right] \exp \left(-i \frac{\zeta}{2 \kappa}\right),
\end{aligned}
$$

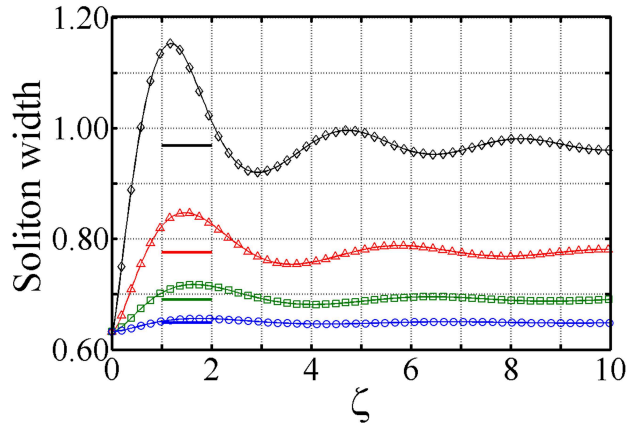

FIG. 12: (color online) Evolution of the boundary (antikink) soliton full width when the initial waveform is given by Eq. (B3) (horizontal bars indicate theoretical predictions). System parameters: $\gamma_{2}=+1, \gamma_{4}=-0.15$, $s=+1, \alpha=1.0, \kappa=1.0 \times 10^{-3}$. Blue circle: $\Omega=4$. Green square: $\Omega=8$. Red triangle: $\Omega=12$. Black diamond:

$$
\Omega=16 \text {. }
$$

where we have written $\mathcal{R}+i \mathcal{I}=i(\mathcal{I}-i \mathcal{R})$ and subsequently dropped the $i$ premultiplier due to the global phase invariance of Eq. (2). Solitons (C4) and (23a) thus have the same intensity distribution (as they must) but they differ in phase by $\pi / 2$ radians [in fact, the phase of solution (C4) is simply $\left.\Psi_{\mathrm{d}}(\tau, \zeta)+\pi / 2\right]$. This form of the cubic-quintic dark soliton has been reported elsewhere in the context of nonlinear-Schrödinger models [31]. It is now straightforward to show that the wellknown " $A-i F$ " representation of the cubic dark soliton emerges in the limit $\left|\gamma_{4}\right| \rho_{0} /\left|\gamma_{2}\right| \ll \mathcal{O}(1)$ [6], where

$$
u_{\mathrm{d}}(\tau, \zeta) \propto A \tanh \left[\left(\gamma_{2} \rho_{0}\right)^{1 / 2} A \Theta_{\mathrm{d}}(\tau, \zeta)\right]-i F
$$

[1] T. Dauxois and M. Peyrard, Physics of Solitons (Cambridge University Press, Cambridge, 2006).

R. K. Dodd, J. C. Eilbeck, J. D. Gibbon, and H. C. Morris, Solitons and Nonlinear Wave Equations (Academic Press, London, 1982).

G. L. Lamb, Elements of Soliton Theory (John Wiley and Sons, New York, 1980).

[2] Y. S. Kivshar and B. A. Malomed, Rev. Mod. Phys. 61, 763 (1989).

V. G. Makhankov, Phys. Rep. 35, 1 (1978)

A. C. Scott, F. Y. F. Chu, and D. W. McLaughlin, Proc. IEEE 61, 1443 (1973).

[3] R. K. Bullough, P. M. Jack, P. W. Kitchenside, and R. Saunders, Phys. Scr. 20, 364 (1979).

E. A. Kuznetsov, A. M. Rubenchik, and V. E. Zakharov, Phys. Rep. 142, 103 (1986).

A. R. Bishop, Phys. Scr. 20, 409 (1979).

P. S. Lomdahl, S. P. Layne, and I. J. Bigio, Los Alamos Science 10, 1 (1984).

A. S. Davydov, Phys. Scr. 20, 387 (1979).

L. D. Faddeev and V. E. Korepin, Phys. Rep. 42, 1 (1978).

R. K. Bullough, in Interaction of Radiation with Condensed Matter, Vol. 1 (International Atomic Energy Agency, Vienna, 1977) p. 381

[4] J. M. Christian, G. S. McDonald, T. F. Hodgkinson, and P. Chamorro-Posada, Phys. Rev. Lett. 108, 034101 (2012).
[5] J. M. Christian, G. S. McDonald, T. F. Hodgkinson, and P. Chamorro-Posada, Phys. Rev. A 86, 023838 (2012).

[6] J. M. Christian, G. S. McDonald, T. F. Hodgkinson, and P. Chamorro-Posada, Phys. Rev. A 86, 023839 (2012).

[7] M. A. Porras, J. Opt. Soc. Am. B 16, 1468 (1999). G. Fibich and G. C. Papanicolaou, Opt. Lett. 22, 1379 (1997).

J. R. Rothenberg, Opt. Lett. 17, 1340 (1992).

[8] J. D. Jackson, Classical Electrodynamics, 3rd ed. (John Wiley and Sons, New York, 1999).

H. Goldstein, Classical Mechanics, 2nd ed. (AddisonWesley, Philippines, 1980).

[9] R. W. Boyd, Nonlinear Optics, 2nd ed. (Academic Press, San Diego, 2003).

[10] Y. S. Kivshar, Opt. Quantum Electron. 30, 571 (1998). Y. S. Kivshar and B. Luther-Davies, Phys. Rep. 298, 81 (1998).

[11] V. E. Zakharov and A. B. Shabat, Sov. Phys. JETP 34, 62 (1972).

V. E. Zakharov and A. B. Shabat, Sov. Phys. JETP 37, 823 (1973).

[12] A. Hasegawa and F. Tappert, Appl. Phys. Lett. 23, 142 (1972).

A. Hasegawa and F. Tappert, Appl. Phys. Lett. 23, 171 (1972).

[13] L. F. Mollenauer, R. H. Stolen, and J. P. Gordon, Phys. Rev. Lett. 45, 1095 (1980).

A. M. Weiner, J. P. Heritage, R. J. Hawkins, R. N. 
Thurston, and E. M. Kirschner, Phys. Rev. Lett. 61, 2445 (1988).

P. Emplit, J. P. Hamaide, F. Reynaud, C. Frohly, and A. Barthelemy, Opt. Commun. 62, 374 (1987).

[14] F. Biancalana and C. Creatore, Opt. Express 16, 14822 (2008).

[15] V. M. Agranovich and V. L Ginzburg, Crystal Optics with Spatial Dispersion, and Excitons (Springer, Berlin, 1984).

[16] K. I. Pushkarov, D. I. Pushkarov, and I. V. Tomov, Opt. Quantum Electron. 11, 471 (1979).

K. I. Pushkarov and D. I. Pushkarov, Rep. Math. Phys. 17, 37 (1980).

[17] E. L. Falcao-Filho, C. B. de Araújo, G. Boudebs, H. Leblond, and V. Sharka, Phys. Rev. Lett. 110, 013901 (2013).

[18] K. Ekvall, C. Lundevall, and P. van der Meulen, Opt. Lett. 26, 896 (2001).

[19] G. I. Stegeman, A. Villeneuve, J. S. Aitchison, and C. N. Ironside, in Fabrication, Properties and Applications of Low-Dimensional Semiconductors, NATO ASI Series 3. High Technology Vol. 3, edited by M. Balkanski and I. Yantchev (Springer, Netherlands, 1995) p. 415.

[20] H. Michinel, Opt. Quantum Electron. 28, 1013 (1996). W. Schmid and T. Vogtman and M Schwoerer, Opt. Commun. 121, 55 (1995).

[21] L. H. Acioli, A. S. L. Gomes, J. M. Hickmann, and C. B. de Araújo, Appl. Phys. Lett. 56, 2279 (1990).

P. Roussignol, D. Ricard, J. Lukasik, and C. Flytzanis, J. Opt. Soc. Am. B 4, 5 (1987).

[22] B. L. Lawrence and G. I. Stegeman, Opt. Lett. 23, 591 (1998).

B. L. Lawrence, M. Cha, W. E. Torruellas, G. I. Stegeman, S. Etemad, G. Baker, and F. Kajzar, Appl. Phys. Lett. 64, 2773 (1994).

B. Lawrence, W. E. Torruellas, M. Cha, M. L. Sundheimer, G. I. Stegeman, J. Meth, S. Etemad, and G. Baker, Phys. Rev. Lett. 73, 597 (1994).

[23] K. Ogusu, Y. Yamasaki, S. Maeda, M. Kitao, and M. Minakata, Opt. Lett. 29, 265 (2004).

G. Boudebs, S. Cherukulappurath, H. Leblond, J. Troles, F. Smektala, and F. Sanchez, Opt. Commun. 219, 427 (2003).

F. Smektala and C. Quemard and V. Couderc and A. Barthelemy, J. Non-Cryst. Solids 274, 232 (2000).

[24] C. Zhan, D. Zhang, D. Zhu, D. Wang, Y. Li, Z. Lu, L. Zhao, and Y. Nie, J. Opt. Soc. Am. B 19, 369 (2002).

[25] G. Stegeman, D. G. Papazoglou, R. Boyd, and S. Tzortzakis, Opt. Express 19, 6387 (2011).

[26] V. Besse, H. Leblond, and G. Boudebs, Phys. Rev. A 92, 013818 (2015).

[27] S. Gatz and J. Herrmann, IEEE J. Quantum Electron. 28, 1732 (1992).

J. Herrmann, Opt. Commun. 87, 161 (1992).

[28] J. Herrmann, Opt. Commun. 91, 337 (1992).

[29] L. Gagnon, J. Opt. Soc. Am. A 6, 1477 (1989).

[30] C. Hua and K. Li, Chaos, Solitons and Fractals 25, 1169 (2005).

S. Tanev and D. I. Pushkarov, Opt. Commun. 141, 322 (1997).

D. Pushkarov and S. Tanev, Opt. Commun. 24, 354 (1996).

Y. S. Kivshar, V. V. Afansjev, and A. W. Snyder, Opt. Commun. 126, 348 (1996).
C. Zhou, X. T. He, and S. Chen, Phys. Rev. A 46, 2277 (1992).

[31] W. Chao, J. Opt. Soc. Am. B 18, 1351 (2001).

W. Chao, Opt. Commun. 187, 415 (2001).

W. Chao, Opt. Commun. 175, 239 (2000).

W. S. Kim and H. T. Moon, Phys. Lett. A 266, 364 (2000).

W. S. Kim and H. T. Moon, J. Korean Phys. Soc. 38, 558 (2001).

Y. Chen, Phys. Rev. E 55, 1221 (1997).

[32] R. W. Micallef, V. V. Afanasjev, Y. S. Kivshar, and J. D. Love, Phys. Rev. E 54, 2936 (1996).

K. Hayata and M. Koshiba, Phys. Rev. E 51, 1499 (1995).

[33] K. J. Blow and N. J. Doran, in Nonlinear Waves in Solid State Physics, NATO ADI Series B: Physics, Vol. 247, edited by A. D. Boardman, M. Bertolotti, and T. Twardowski (Plenum Press, New York, 1990) p. 325.

[34] J. M. Christian, G. S. McDonald, and P. ChamorroPosada, Phys. Rev. A 76, 033833 (2007).

[35] J. M. Christian, G. S. McDonald, and P. ChamorroPosada, Phys. Rev. A 81, 053831 (2010).

[36] S. Blair, Chaos, 10, 570 (2000).

K. Marinov, D. I. Pushkarov, and A. Shivarova, in Soliton-Driven Photonics, edited by A. D. Boardman and A. P. Sukhorukov (Kluwer Academic Press, Netherlands, 2001) p. 95.

C. Chen and S. Chi, Opt. Commun. 157, 170 (1998).

[37] A. E. Kaplan, Phys. Rev. Lett 55, 1291 (1985).

A. E. Kaplan, IEEE J. Quantum Electron. QE-21, 1538 (1985).

A. E. Kaplan and C. T. Law, IEEE J. Quantum Electron. QE-21, 1529 (1985).

[38] P. Chamorro-Posada, G. S. McDonald, and G. H. C. New, Opt. Commun. 192, 1 (2001).

[39] M. G. Vakhitov and A. A. Kolokolov, Radiophys. Quantum Electron. 16, 783 (1973).

[40] J. M. Christian and G. S. McDonald and P. ChamorroPosada, J. Phys. A: Math. Theor. 43, 085212 (2010).

J. M. Christian, G. S. McDonald, and P. ChamorroPosada, J. Phys. A: Math. Theor. 40, 1545 (2007).

J. M. Christian, G. S. McDonald, R. J. Potton, and P. Chamorro-Posada, Phys. Rev. A 76, 033834 (2007).

[41] J. Satsuma and N. Yajima, Suppl. Prog. Theor. Phys. 55, 284 (1974).

[42] D. E. Pelinovsky, V. V. Afanasjev, and Y. S. Kivshar, Phys. Rev. E 53, 1940 (1996).

[43] N. Akhmediev, A. Ankiewicz, and R. Grimshaw, Phys. Rev. E 59, 6088 (1999).

[44] I. V. Barashenkov, Phys. Rev. Lett. 77, 1193 (1996).

[45] D. E. Pelinovsky, Y. S. Kivshar, and V. V. Afanasjev, Phys. Rev. A 54, 2015 (1996).

[46] J. M. Christian, G. S. McDonald, M. J. Lundie, and A. Kotsampaseris, companion article submitted to Physical Review A (2018).

[47] M. Crosta, A. Fratalocchi, and S. Trillo, Phys. Rev. A 84, 063809 (2011).

Y. S. Kivshar and V. V. Afansjev and A. W. Snyder, Opt. Commun. 126, 348 (1996).

[48] B. Kibler, J. Fatome, C. Finot, G. Millot, G. Genty, B. Wetzel, N. Akhmediev, F. Dias, and J. M. Dudley, Sci. Rep. 2, 463 (2012).

B. Kibler, J. Fatome, C. Finot, G. Millot, F. Dias, G. Genty, N. Akhmediev, and J. M. Dudley, Nature Physics 
6, 790 (2010).

D. H. Peregrine, J. Aust. Math. Soc. Ser. B 25, 16 (1983).

[49] S. Toenger, T. Godin, C. Billet, F. Dias, M. Erkintalo, G. Genty, and J. M. Dudley, Sci. Rep. 5, 10380 (2015).

N. Akhmediev, J. M. Soto-Crespo, and A. Ankiewicz, Phys. Lett. A 373, 2137 (2009).

N. Akhmediev, A. Ankiewicz, and M. Taki, Phys. Lett. A 373, 675 (2009).

[50] A. Chabchoub, N. P. Hoffmann, M. Onorato, G. Genty, J. M. Dudley, and N. Akhmediev, Phys. Rev. Lett. 111,
054101 (2013).

A. Chabchoub, N. P. Hoffmann, and N. Akhmediev, Phys. Rev. Lett. 106, 204502 (2011).

[51] N. Horiuchi, Nature Photonics 8, 499 (2014).

J. Fatome, C. Finot, G. Millot, A. Armaroli, and S. Trillo, Phys. Rev. X 4, 021022 (2014).

B. Wetzel and A. Pasquazi and R. Morandotti, Physics 7, 48 (2014). 